\title{
Image Restoration and Decomposition via Bounded Total Variation and Negative Hilbert-Sobolev Spaces
}

\author{
Linh H. Lieu • Luminita A. Vese
}

Published online: 14 May 2008

(C) Springer Science+Business Media, LLC 2008

\begin{abstract}
We propose a new class of models for image restoration and decomposition by functional minimization. Following ideas of Y. Meyer in a total variation minimization framework of L. Rudin, S. Osher, and E. Fatemi, our model decomposes a given (degraded or textured) image $u_{0}$ into a sum $u+v$. Here $u \in B V$ is a function of bounded variation (a cartoon component), while the noisy (or textured) component $v$ is modeled by tempered distributions belonging to the negative Hilbert-Sobolev space $H^{-s}$. The proposed models can be seen as generalizations of a model proposed by S. Osher, A. Solé, L. Vese and have been also motivated by D. Mumford and B. Gidas. We present existence, uniqueness and two characterizations of minimizers using duality and the notion of convex functions of measures with linear growth, following I. Ekeland and R. Temam, F. Demengel and R. Temam. We also give a numerical algorithm for solving the minimization problem, and we present numerical results of denoising, deblurring, and decompositions of both synthetic and real images.
\end{abstract}

Keywords Functional minimization - Functions of bounded variation - Negative Hilbert-Sobolev spaces · Duality · Image restoration · Image decomposition · Image deblurring · Image analysis · Fourier transform

\section{Introduction and Motivations}

Image restoration and decomposition are of important interest in image processing. In the restoration case, one seeks to recover a "true" image $u$ from an observed, often

L.H. Lieu $(\bowtie)$

Department of Mathematics, University of California, Davis, One Shields Avenue, Davis, CA 95616, USA

e-mail: 1lieu@math.ucdavis.edu

\section{L.A. Vese}

Department of Mathematics, University of California, Los Angeles, 405 Hilgard Avenue, Los Angeles, CA 90095-1555, USA

e-mail: 1vese@math.ucla.edu 
noisy and/or blurry, image $u_{0}$. Many well-known models for image restoration work by decomposing the observed image $u_{0}$ into a sum of two components, $u_{0}=(u+v) \in$ $L^{2}$, in a variational approach, where $u$ is a piecewise smooth geometric component representing the objects in the image and $v$ is the oscillatory component representing the noise. Examples include S. Geman-D. Geman [19], D. Mumford, J. Shah [26], among others. One such model is proposed by Rudin, Osher, and Fatemi [30, 31]:

$$
\inf _{u \in B V(\Omega)}\left\{\lambda \int_{\Omega}|D u|+\int_{\Omega}\left|u_{0}-u\right|^{2} d x\right\} .
$$

Here $\Omega$ is an open, bounded and connected set in the plane, with Lipschitz boundary $\partial \Omega$. The first term in (1) is called the Total Variation (TV) or regularization term, and the second the fidelity (fitting) term. $\lambda$ is a positive (tuning) parameter. The infimum is taken over all functions in $B V(\Omega)$ (functions of bounded variations). The solution to (1) is the geometric component $u$ in the decomposition $u_{0}=u+v$. Here, the $v$ component is written as $u_{0}-u$. Existence and uniqueness results of this minimization problem can be found in $[1,3,10]$, or in [35] for a more general case.

The model (1) performs well for removing noise while preserving edges. However, it fails to separate well oscillatory components from high-frequencies components. For example, edges of an object are high-frequency components and noise is an oscillatory component. Both the $u$ and $v$ components in the ROF model contain high-frequencies component. That is, the $v$ component not only contains oscillatory components, it also contains high-frequency components. One can see shadows of edges in the noise/textured component $v$, even when the parameter $\lambda$ is not so small. Alternatively, to remedy this situation, Y. Meyer suggested to replace the $L^{2}$-norm in the fidelity term of (1) with a weaker norm more appropriate for modeling textured or oscillatory patterns. He proposed the following minimization problem [24]:

$$
\inf _{u \in B V(\Omega)}\left\{\lambda \int_{\Omega}|D u|+\left\|u_{0}-u\right\|_{*}\right\},
$$

where the $\|\cdot\|_{*}$-norm is defined as follows:

Definition 1.1 Let $\mathrm{G}$ denote the Banach space consisting of all generalized functions $f$ which can be written as $f=\operatorname{div}(\vec{g}), \vec{g}=\left(g_{1}, g_{2}\right), g_{1}, g_{2} \in L^{\infty}(\Omega)$, induced by the norm $\|f\|_{*}=\inf _{f=\operatorname{div}(\vec{g})}\left\|\sqrt{g_{1}^{2}+g_{2}^{2}}\right\|_{L^{\infty}(\Omega)}$.

The space $G$ can be identified with $\dot{W}^{-1, \infty}(\Omega)$, the dual of the homogeneous space $\dot{W}^{1,1}(\Omega):=\left\{f \in L^{1}(\Omega): \nabla f \in L^{1}(\Omega)^{2}\right\}$. In [24], Y. Meyer also proposes two other spaces for modeling oscillations, denoted by $F$ and $E$. The space $F$ is defined as $G$, but instead of $L^{\infty}(\Omega), g_{1}, g_{2}$ belong to the John and Nirenberg space $B M O(\Omega)$. The space $E$ is the generalized Besov space $\dot{B}_{\infty, \infty}^{-1}(\Omega)=\triangle \dot{B}_{\infty, \infty}^{1}(\Omega)$. Oscillatory patterns have smaller norms in $G, F$ and $E$ than in $L^{2}$ (see [24] for details). Thus, in a minimization problem such as (1) and (2), these spaces of generalized functions are more appropriate for decomposing an image into a piecewise smooth (or cartoon) part and an oscillatory (or textured) part than the space $L^{2}$. 
Mumford and Gidas, taking a statistical approach, have proposed in [25] several stochastic models for analysis of generic images. In this paper, the authors showed that white noise lives in $\bigcap_{\varepsilon>0} H_{l o c}^{-1-\varepsilon}$, where $H_{l o c}^{-s}$ (for any $s \geq 0$ ) is the HilbertSobolev space of negative degree of differentiability. They also show that if we model images as samples from a scale and translation invariant probability measure on $\mathcal{S}^{*}$, then such measure is not supported on the subspace of locally integrable functions. The measure is rather supported on spaces of generalized functions, such as the $\mathrm{H}^{-S}$ spaces, with $s>0$. The work of Alvarez, Gousseau, Morel [2, 21] also supports that natural images are not well represented by the component $u$, a function of bounded variation. Thus, there is a need to represent images with textures by weaker, larger spaces of generalized functions or by distributions. However, this leads to more complicated models like (2), instead of the simpler model (1).

The difficulty with the minimization problem (2) is that it cannot be solved directly due to the form of the $*$-norm. That is to say, the subdifferential of the functional and the associated Euler-Lagrange equation cannot be expressed directly with respect to $u$. For a practical alternative, the authors in [36] have introduced the following minimization problem as an approximation to (2):

$$
\begin{aligned}
\inf _{u \in B V(\Omega), g_{1}, g_{2} \in L^{p}(\Omega)^{2}} G_{p}(u, \vec{g})= & \lambda \int_{\Omega}|D u|+\mu \int_{\Omega}\left|u_{0}-(u+\operatorname{div} \vec{g})\right|^{2} d x \\
+ & {\left[\int_{\Omega}\left(\sqrt{g_{1}^{2}+g_{2}^{2}}\right)^{p} d x\right]^{\frac{1}{p}}, }
\end{aligned}
$$

where $\lambda, \mu>0$ are tuning parameters, and $p \geq 1$. The first term in (3) ensures that $u \in B V(\Omega)$, the second term ensures that $v=u_{0}-u \approx \operatorname{div}(\vec{g})$, and the last term ensures that $g_{i} \in L^{p}(\Omega)$. The minimization (3) is an approximation to (2), by taking $\mu, p \rightarrow \infty$ (in the limit, $u_{0}-u=\operatorname{div}(\vec{g})$ a.e., for all those $\vec{g}$ with smallest $L^{\infty}(\Omega)$ norm, the middle term disappears and the last term becomes $\left.\left\|u_{0}-u\right\|_{*}\right)$. In the case $p=2, v=\operatorname{div} \vec{g}$ in (3) also corresponds to $v \in \dot{H}^{-1}(\Omega)$, the dual of the space $\dot{H}^{1}(\Omega)$. Indeed, for $v \in \dot{H}^{-1}(\Omega)$, there is a $P \in \dot{H}^{1}(\Omega)$ such that

$$
\|v\|_{\dot{H}^{-1}(\Omega)}^{2}=\inf _{g_{1}, g_{2} \in L^{2}(\Omega), v=\operatorname{div} \vec{g}} \int_{\Omega}\left(g_{1}^{2}+g_{2}^{2}\right) d x=\int_{\Omega}|\nabla P|^{2} d x,
$$

that is to say, $v=\operatorname{div}(\nabla P)=\triangle P$, or equivalently, $P=\triangle^{-1} v$ (compare with the space $F$ proposed by Y. Meyer to model textures).

Limiting to the case $p=2$ and using the inverse Laplacian, the authors in [27] have simplified the minimization problem (3) in a new problem corresponding to the case $\mu=\infty$ in (3). They proposed the following:

$$
\inf _{u \in B V(\Omega)}\left\{\lambda \int_{\Omega}|D u|+\left|u_{0}-u\right|_{\dot{H}^{-1}(\Omega)}^{2}\right\},
$$

where $|v|_{\dot{H}^{-1}(\Omega)}^{2}=\int_{\Omega}\left|\nabla \Delta^{-1} v\right|^{2} d x$, which is a semi-norm dual to the semi-norm $|u|_{H^{1}(\Omega)}:=\int_{\Omega}|\nabla u|^{2} d x$ of $H^{1}(\Omega)$. This model yields an exact decomposition $u_{0}=$ $u+v$, with $u \in B V(\Omega)$ and $v \in \dot{H}^{-1}(\Omega)$. Solving (4) leads to solving an equivalent 
non-linear fourth order Euler-Lagrange PDE with a severe CFL condition (for details see [27]). Numerical experiments show that the models (3) and (4) separate texture from objects better than the ROF model.

Motivated by [24, 27, 36], J.-F. Aujol and collaborators have proposed related approximations to Meyer's $(B V, G)$ model in the interesting and inspiring work [5, 79]. In particular in [9], Aujol and Chambolle consider Sobolev spaces of negative exponent, as in [36]. They make a statistical analysis of the Gaussian white noise regarding the choice of the norm, and they propose a decomposition model $u_{0}=u+v+w$, with $u \in B V$ the cartoon component, $v \in W^{-1, p}$ the texture component $(p \in[1, \infty])$, and $w \in E$ the noise component. They favor the choice of the $E$ space to model white Gaussian noise, rather then the $H^{-1}$ norm, and the $G$ space to model texture. A related elegant work has been proposed by I. Daubechies and G. Teschke [11, 12] in a wavelets approach. In $[11,12]$, the authors have modified the Vese-Osher and OsherSolé-Vese energies in (3) and (4) by replacing $B V(\Omega)$ with the space $B_{1}^{1}\left(L^{1}(\Omega)\right)$ in the regularizing term, and limiting themselves to the case $p=2$, whereby they have arrived to a new minimization problem which gives a decomposition $u_{0} \approx u+v$, with $u \in B_{1}^{1}\left(L^{1}(\Omega)\right)$ and $v \in H^{-1}(\Omega)$.

Other interesting related work for image decomposition and cartoon and texture separation using generalized functions and dual spaces are by Starck, Elad and Donoho [34] using wavelets dictionaries, Tadmor, Nezzar and Vese [33] for a hierarchical $\left(B V, L^{2}\right)$ decomposition, Esedoglu and Osher [16], Levine [23] for dual functionals, Le-Vese [22] for $(B V, F)$ decompositions, Garnett, Le, Meyer, Vese [18] for $\left(B V, \dot{B}_{p, q}^{-\alpha}\right)$ decompositions, among others.

Motivated by the works of Meyer [24], Osher-Solé-Vese [27], MumfordGidas [25], we propose in this paper a new convex minimization problem (or rather, a class of convex minimization problems), in which we decompose a given image into $u_{0}=u+v$ where $u$ is of bounded variation and $v$ belongs to one of the HilbertSobolev spaces of negative degree of differentiability.

The precise formulation of our proposed model is given in Sect. 2. As we shall show below, solving the proposed convex minimization problem with respect to the unknown $u$ leads to solving a second-order Euler-Lagrange PDE, instead of a fourthorder PDE [27]. Moreover, the new model is more general than (1) and (4). In fact, it recovers (1) when we set $s=0$, and it becomes an equivalent form of (4) when we make simple modification in the form of the norm $H^{-s}$. A related preliminary work inspired from model [27] and also using the Fourier Transform for computations and variants is by S. Roudenko [29].

The plan of this paper is as follows: in Sect. 2, we give a detailed description of the proposed model. Existence and uniqueness of solutions and two characterizations of minimizers are given in Sects. 3 and 4, respectively. In Sect. 5 we give detailed explanation to our numerical algorithm and implementation of our model. Then in Sect. 6, we give numerical results and comparisons of these with other models. 


\section{Description of the New Model}

2.1 The Space $H^{s}\left(\mathbb{R}^{n}\right)[13]$

Let $\mathcal{S}\left(\mathbb{R}^{n}\right):=\left\{u \in \mathcal{C}^{\infty}\left(\mathbb{R}^{n}\right): \forall \alpha \in \mathbb{N}, \beta \in \mathbb{N}^{n},|x|^{\alpha}\left|D^{\beta} u\right| \rightarrow 0\right.$ as $\left.|x| \rightarrow+\infty\right\}$, the Schwartz space. The dual $\mathcal{S}\left(\mathbb{R}^{n}\right)^{*}$ to $\mathcal{S}\left(\mathbb{R}^{n}\right)$ is the space of tempered distributions. Recall that the Fourier Transform of a function $f \in \mathcal{S}\left(\mathbb{R}^{n}\right)$, or of $f \in L^{1}\left(\mathbb{R}^{n}\right)$, denoted $\hat{f}$, is given by

$$
\mathfrak{F} f(\xi):=\hat{f}(\xi) \stackrel{\text { def }}{=} \int_{\mathbb{R}^{n}} f(x) e^{-2 \pi i x \cdot \xi} d x, \quad \xi \in \mathbb{R}^{n} .
$$

Let the bracket $\langle$,$\rangle denote the duality pairing between \mathcal{S}\left(\mathbb{R}^{n}\right)^{*}$ and $\mathcal{S}\left(\mathbb{R}^{n}\right)$. The Fourier Transform of a tempered distribution $g$ is the tempered distribution $\hat{g}$ defined by:

$$
\langle\hat{g}, \varphi\rangle=\langle g, \hat{\varphi}\rangle \quad \text { for all } \varphi \in \mathcal{S}\left(\mathbb{R}^{n}\right)
$$

Definition 2.1 For any $s \in \mathbb{R}$, the space $H^{s}\left(\mathbb{R}^{n}\right)$ consists of tempered distributions $g$ such that $\left(1+|\xi|^{2}\right)^{s / 2} \cdot \hat{g} \in L^{2}\left(\mathbb{R}^{n}\right)$, where $\hat{g}$ is the Fourier transform of $g$.

The space $H^{s}\left(\mathbb{R}^{n}\right)$ is a Hilbert space equipped with the inner product

$$
\langle f, g\rangle_{s}=\int_{\mathbb{R}^{n}}\left(1+|\xi|^{2}\right)^{s} \hat{f}(\xi) \overline{\hat{g}(\xi)} d \xi
$$

and the associated norm $\|f\|_{s}=\sqrt{\langle f, f\rangle_{s}}=\left(\int_{\mathbb{R}^{n}}\left(1+|\xi|^{2}\right)^{s}|\hat{f}(\xi)|^{2} d \xi\right)^{1 / 2}$. When $s=m$ is an integer, then $H^{s}\left(\mathbb{R}^{n}\right)$ is the same as the Sobolev Space $H^{m}\left(\mathbb{R}^{n}\right)$ with equivalent norms. The dual to $H^{-s}\left(\mathbb{R}^{n}\right)$ is the space $H^{s}\left(\mathbb{R}^{n}\right)$. Observe that if $s_{1}>$ $s_{2} \geq 0$, then $\|f\|_{s_{1}}>\|f\|_{s_{2}}$. Thus we have the following continuous embeddings (injections) of spaces

$$
\begin{aligned}
& \mathcal{S}\left(\mathbb{R}^{n}\right) \subset H^{s_{1}}\left(\mathbb{R}^{n}\right) \subset H^{s_{2}}\left(\mathbb{R}^{n}\right) \subset \cdots \subset H^{0}\left(\mathbb{R}^{n}\right) \\
& \quad=L^{2}\left(\mathbb{R}^{n}\right) \subset \cdots \subset H^{-s_{2}}\left(\mathbb{R}^{n}\right) \subset H^{-s_{1}}\left(\mathbb{R}^{n}\right) \subset \mathcal{S}\left(\mathbb{R}^{n}\right)^{*} .
\end{aligned}
$$

\subsection{Extending from a Bounded Domain $\Omega$ to $\mathbb{R}^{2}$}

When working with images, we are dealing with functions defined only on a bounded domain. On the other hand, the $H^{s}$ norm is given via the Fourier Transform which is defined for functions whose values on the whole space are known. To resolve this, we will consider extending a function given on a bounded domain $\Omega \subset \mathbb{R}^{2}$ by zeros to the whole $\mathbb{R}^{2}$ space. It is clear that extension by zeros is a continuous embedding of $L^{2}(\Omega)$ into $L^{2}\left(\mathbb{R}^{2}\right)$. Moreover, it is an embedding of $B V(\Omega)$ into $B V\left(\mathbb{R}^{2}\right)$ [17]. In addition, since $\partial \Omega$ is Lipschitz, Poincaré-Wirtinger Inequality [17] implies that $B V(\Omega)$ is continuously embedded into $L^{2}(\Omega)$. We thus have the following continuous embeddings: $B V(\Omega) \subset L^{2}(\Omega) \subset L^{2}\left(\mathbb{R}^{2}\right) \subset H^{-s}\left(\mathbb{R}^{2}\right), s \geq 0$.

Henceforth in this paper in the continuous case, when the functions are defined only on $\Omega \subset \mathbb{R}^{2}, \partial \Omega$ is Lipschitz, we shall use extension by zeros to compute the 
$H^{-s}$ norm. We will consider a function $f: \Omega \rightarrow \mathbb{R}$ to be in $H^{-s}\left(\mathbb{R}^{2}\right), s>0$, if after extending by zeros to $\mathbb{R}^{2}$, the extended function, which we will also refer to as $f$, belongs to $H^{-s}\left(\mathbb{R}^{2}\right)$. In the discrete case (see Sect. 5), we use the Discrete Fourier Transform (DFT) and the Inverse Discrete Fourier Transform (IDFT), where the explicit extension by zeros of discrete functions outside $\bar{\Omega}$ is no longer needed.

\subsection{The New Model}

Hereafter, $s$ will always be positive, and we shall use the notation $L^{2}$ and $H^{-s}$ for $L^{2}\left(\mathbb{R}^{2}\right)$ and $H^{-s}\left(\mathbb{R}^{2}\right)$, respectively. We propose the following new variational model for image restoration and decomposition:

$$
\inf _{u \in B V(\Omega)} F(u):=\lambda|u|_{B V(\Omega)}+\left\|u_{0}-K u\right\|_{-s}^{2},
$$

where $s \geq 0$ is a parameter for the fidelity norm, $\Omega$ is an open, bounded and connected subset of $\mathbb{R}^{2}$ with Lipschitz boundary, $\lambda>0$ is a regularization parameter, $|u|_{B V(\Omega)}:=\int_{\Omega}|D u|$ is the regularization (total variation) term, $\left\|u_{0}-K u\right\|_{-s}^{2}=$ $\int_{\mathbb{R}^{2}}\left(1+|\xi|^{2}\right)^{-s}\left|\hat{u}_{0}-\widehat{K u}\right|^{2} d \xi$ is the fidelity term. We assume that the operator $K$ is an injective continuous linear operator from $L^{2}$ into $L^{2}$ such that $K 1_{\Omega} \neq 0$.

\section{Remark 2.1}

(i) If $K$ to is the identity operator and $s=0$, by Parceval Identity $\int|g|^{2} d x=$ $\int|\hat{g}|^{2} d \xi$, (5) becomes exactly (1), i.e. our proposed model recovers the ROF model.

(ii) We can obtain an equivalent form of (4) from (5) by setting $s=1$ and writing $\left\|u_{0}-u\right\|_{H_{0}^{-s}}:=\int|\xi|^{-2 s}\left|\hat{u}_{0}-\hat{u}\right|^{2} d \xi$, that is, we change the $H^{-s}$ norm into a semi-norm by removing 1 from the first term in the integrand. Thus, we obtain an equivalent formulation of the OSV model [27].

\section{Existence and Uniqueness of Solutions}

We now prove existence and uniqueness of minimizers for the proposed model, adapting the techniques from $[1,10,35]$ to the $\left(B V, H^{-s}\right)$ case.

Theorem 3.1 Given $\Omega \subset \mathbb{R}^{2}$, open, bounded and connected, with Lipschitz boundary, $u_{0} \in H^{-s}, u_{0}=0$ outside of $\bar{\Omega}, \lambda>0$, and $K: L^{2} \rightarrow L^{2}$ an injective continuous linear operator such that $K 1_{\Omega} \neq 0$, then the minimization problem

$$
\inf _{u \in B V(\Omega)} F(u)=\lambda|u|_{B V(\Omega)}+\left\|u_{0}-K u\right\|_{-s}^{2}, \quad s>0,
$$

has a unique solution in $B V(\Omega)$.

Proof Note the infimum of $F(u)$ is finite. Let $u_{n} \in B V(\Omega)$ be a minimizing sequence of $F$. Then there exists a constant $M>0$ for which $\left|u_{n}\right|_{B V(\Omega)} \leq M$ and 
$\left\|u_{0}-K u_{n}\right\|_{-s}^{2} \leq M$ for all $n \geq 0$. By the Poincaré-Wirtinger inequality, we have a constant $C>0$ which depends only on $\Omega$ such that

$$
\left\|u_{n}-\frac{1}{|\Omega|} \int_{\Omega} u_{n}\right\|_{L^{2}(\Omega)} \leq C\left|u_{n}\right|_{B V(\Omega)} \leq C M, \text { for all } n .
$$

First we show that there is $C^{\prime}>0$ such that $\frac{1}{|\Omega|}\left|\int_{\Omega} u_{n}\right| \leq C^{\prime}$, for all $n \geq 0$. Following $[1,35]$, denote by $w_{n}=\left(\frac{1}{|\Omega|}\left|\int_{\Omega} u_{n}\right|\right) \chi_{\Omega}$ and $v_{n}=u_{n}-w_{n}$. Then clearly $w_{n}, v_{n} \in$ $B V(\Omega)$. Moreover, we have from (6)

$$
\left\|v_{n}\right\|_{-s} \leq\left\|v_{n}\right\|_{L^{2}}=\left\|u_{n}-\frac{1}{|\Omega|} \int_{\Omega} u_{n}\right\|_{L^{2}(\Omega)} \leq C,
$$

for some constant $C>0$ independent of $n$, for all $n$. Thus, since

$$
M \geq\left\|u_{0}-K u_{n}\right\|_{-s}^{2}=\left\|u_{0}-K v_{n}-K w_{n}\right\|_{-s}^{2},
$$

$u_{0}$ and $K v_{n}$ are uniformly bounded in norm, $K$ is a bounded operator, we deduce $\left\|K w_{n}\right\|_{-s} \leq M^{\prime}$ uniformly. $C^{\prime}$.

This implies $\frac{1}{|\Omega|}\left|\int_{\Omega} u_{n}\right|\left\|K \chi_{\Omega}\right\|_{-s}=\left\|K w_{n}\right\|_{-s} \leq M^{\prime \prime}$ for all $n$, or $\frac{1}{|\Omega|}\left|\int_{\Omega} u_{n}\right| \leq$

Thus, for all $n \geq 0,\left\|u_{n}\right\|_{L^{2}(\Omega)} \leq M^{\prime}$, implying $\left\|u_{n}\right\|_{L^{1}(\Omega)} \leq M^{\prime}$. Hence uniformly

$$
\left\|u_{n}\right\|_{B V(\Omega)}:=\left\|u_{n}\right\|_{L^{1}(\Omega)}+\left|u_{n}\right|_{B V(\Omega)} \leq M^{\prime \prime} .
$$

Therefore there exists a subsequence, still denoted $u_{n}$, and a $u \in B V(\Omega)$ such that $u_{n} \rightarrow u$ in $L^{1}(\Omega)$ and

$$
|u|_{B V(\Omega)} \leq \liminf _{n \rightarrow \infty}\left|u_{n}\right|_{B V(\Omega)} .
$$

Moreover, by passing to a subsequence if necessary, $u_{n} \rightarrow u$ weakly in $L^{2}(\Omega)$. After extending $u_{n}, u$ by zeros to $\mathbb{R}^{2}$, we still have $u_{n} \rightarrow u$ weakly in $L^{2}$. Since $K$ is a continuous linear operator from $L^{2}$ to $L^{2}, \int_{\mathbb{R}^{2}} K u_{n}(x) \varphi(x) d x=$ $\int_{\mathbb{R}^{2}} u_{n}(x) K^{*} \varphi(x) d x, \forall \varphi \in L^{2}$. Therefore, $K u_{n} \rightarrow K u$ weakly in $L^{2}$.

To show $K u_{n} \rightarrow K u$ weakly in $H^{-s}$, we recall that for any $\varphi \in H^{-s}, \frac{\hat{\varphi}(\xi)}{\left(1+|\xi|^{2}\right)^{s / 2}} \in$ $L^{2}\left(\mathbb{R}^{2} ; \mathbb{C}\right)$, therefore $\frac{\hat{\varphi}(\xi)}{\left(1+|\xi|^{2}\right)^{s}} \in L^{2}\left(\mathbb{R}^{2} ; \mathbb{C}\right)$. So $\mathfrak{F}\left(\frac{\hat{\varphi}(\xi)}{\left(1+|\xi|^{2}\right)^{s}}\right) \in L^{2}\left(\mathbb{R}^{2} ; \mathbb{C}\right)$. Hence (by subsequently taking the real part and then the imaginary part and combining them afterwards)

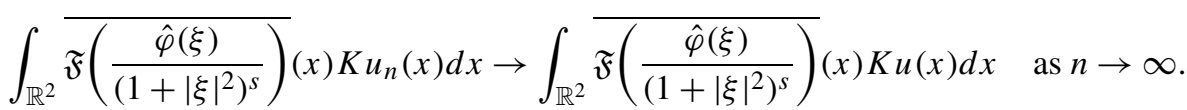

Applying $\int v \overline{\hat{w}}=\int \overline{\hat{v}} w$, we thus have for any $\varphi \in H^{-s}$,

$$
\int_{\mathbb{R}^{2}} \frac{\hat{\varphi} \widehat{\widehat{K u_{n}}}}{\left(1+|\xi|^{2}\right)^{s}} d \xi \rightarrow \int_{\mathbb{R}^{2}} \frac{\hat{\varphi} \widehat{\widehat{K u}}}{\left(1+|\xi|^{2}\right)^{s}} d \xi \quad \text { as } n \rightarrow \infty .
$$


Therefore, $u_{0}-K u_{n} \rightarrow u_{0}-K u$ weakly in $H^{-s}$ and by the lower semi-continuity of the $H^{-s}$-norm, we obtain

$$
\left\|u_{0}-K u\right\|_{-s}^{2} \leq \liminf _{n \rightarrow \infty}\left\|u_{0}-K u_{n}\right\|_{-s}^{2} .
$$

Hence,

$$
F(u):=\lambda|u|_{B V(\Omega)}+\left\|u_{0}-K u\right\|_{-s}^{2} \leq \liminf _{n \rightarrow \infty}\left(\lambda\left|u_{n}\right|_{B V(\Omega)}+\left\|u_{0}-K u_{n}\right\|_{-s}^{2}\right),
$$

and $u$ is a minimizer.

The uniqueness of a minimizer $u \in B V(\Omega)$ is an immediate consequence of the strict convexity of $F(u)$ and the assumptions $K$ injective and $K 1_{\Omega} \neq 0$.

Remark 3.1 The above existence and uniqueness result also holds for other regularizing functionals on $B V(\Omega)$ instead of the total variation. For example, $|u|_{B V(\Omega)}$ can be substituted by $\int_{\Omega} \psi(D u)$ defined in the sense of convex functions of measures (see $[14,35]$ ), where $\psi: \mathbb{R}^{2} \rightarrow[0, \infty)$ is continuous, even, convex, and satisfying $\psi(0)=0, a|x|-b \leq \psi(x) \leq a|x|+b$ for some constants $a>0$ and $b \geq 0$, and any $x \in \mathbb{R}^{2}$. Examples are $\psi(x)=\left|x_{1}\right|+\left|x_{2}\right|, \psi(x)=\sqrt{\varepsilon^{2}+|x|^{2}}-|\varepsilon|$, $\psi(x)=\log \cosh \left(\varepsilon^{2}+|x|^{2}\right)$, with $\varepsilon>0$.

\section{Characterizations of Minimizers}

In this section we present two approaches for characterization of the solution of our proposed model. The first approach is to characterize the subdifferential $\partial F$ of the functional $F$ by computing the dual problem to (5) (a simplified version of the subdifferential will be used in our numerical approximation). The second approach is to utilize the $H^{-s}$ inner product to define a new "texture" norm, and thus we illustrate how our proposed model discriminates cartoon versus texture.

\subsection{Dual Problem Formulation and Characterization of Minimizers}

We will show in this section, via characterizations of the subdifferential of $F$, the optimality conditions satisfied by the solution of (5), including a formulation of the boundary conditions. We follow closely the steps in [35], based on the techniques of Demengel-Temam for the problem of minimal surfaces [14] and duality results from Ekeland-Temam [15] and Temam-Strang [32].

For numerical purposes, we consider another well-defined approximate functional $F_{\varepsilon}$ of $F, \varepsilon \geq 0$, defined for $u \in L^{2}(\Omega)$ by $[1,14]$

$$
F_{\varepsilon}(u)= \begin{cases}\int_{\Omega} \psi(|D u|)+\left\|u_{0}-K u\right\|_{-s}^{2}, & \text { if } u \in B V(\Omega), \\ +\infty, & \text { if } u \in L^{2}(\Omega) \backslash B V(\Omega),\end{cases}
$$

where $\psi(z)=\lambda\left(\sqrt{\varepsilon^{2}+|z|^{2}}-|\varepsilon|\right), z \in \mathbb{R}$, and $F_{0}(u)=F(u)$. It can be shown as before that $F_{\varepsilon}$ also has a unique minimizer $u \in B V(\Omega)$ (see also [1, 35]) and we will compute $\partial F_{\varepsilon}(u)$, because $F(u)=\inf _{v \in L^{2}(\Omega)} F(v)$ if and only if $0 \in \partial F(u)$. 
For $u \in L^{2}(\Omega)$, we say that $\phi \in L^{2}(\Omega)^{*}=L^{2}(\Omega)$ satisfies $\phi \in \partial F_{\varepsilon}(u)$ if and only if [15]

$$
\begin{aligned}
F_{\varepsilon}(u) & \in \mathbb{R} \quad \text { and } \quad F_{\varepsilon}(u)-\langle\phi, u\rangle_{L^{2}(\Omega) \times L^{2}(\Omega)} \leq F_{\varepsilon}(v)-\langle\phi, v\rangle_{L^{2}(\Omega) \times L^{2}(\Omega)}, \\
\forall v & \in L^{2}(\Omega) .
\end{aligned}
$$

The Fourier Transform can be written as a sum of two continuous linear operators as

$$
\begin{aligned}
(\mathfrak{F} f)(\xi) & =\left(\mathcal{F}_{C} f\right)(\xi)+i\left(\mathcal{F}_{S} f\right)(\xi) \\
& =\int_{\mathbb{R}^{2}} f(x) \cos (2 \pi x \cdot \xi) d x+i \int_{\mathbb{R}^{2}} f(x) \sin (2 \pi x \cdot \xi) d x .
\end{aligned}
$$

The definitions of $\mathcal{F}_{C}$ and $\mathcal{F}_{S}$ can be extended in a natural way to tempered distributions $f \in H^{-s}$ by $\left\langle\mathcal{F}_{C} f, \varphi\right\rangle=\left\langle f, \mathcal{F}_{C} \varphi\right\rangle,\left\langle\mathcal{F}_{S} f, \varphi\right\rangle=\left\langle f, \mathcal{F}_{S} \varphi\right\rangle$, for all $\varphi \in \mathcal{S}\left(\mathbb{R}^{2}\right)$.

Let $\mathcal{F}_{1}, \mathcal{F}_{2}: L^{2}(\Omega) \rightarrow L^{2}\left(\mathbb{R}^{2}\right)$ be continuous linear operators defined by

$$
\begin{aligned}
& \left(\mathcal{F}_{1} v\right)(y)=\left(1+|y|^{2}\right)^{-s / 2}\left(\mathcal{F}_{C} K v_{\text {ext }}\right)(y), \\
& \left(\mathcal{F}_{2} v\right)(y)=\left(1+|y|^{2}\right)^{-s / 2}\left(\mathcal{F}_{S} K v_{\text {ext }}\right)(y),
\end{aligned}
$$

where $v \in L^{2}(\Omega), v_{\text {ext }}$ denotes the extension by zeros of $v$ from $\bar{\Omega}$ to $\mathbb{R}^{2}$. For the data image $u_{0} \in H^{-s}$, we denote by $\tilde{f}_{1}(y):=\left(1+|y|^{2}\right)^{-s / 2}\left(\mathcal{F}_{C} u_{0}\right)(y)$ and $\tilde{f}_{2}(y):=$ $\left(1+|y|^{2}\right)^{-s / 2}\left(\mathcal{F}_{S} u_{0}\right)(y)$.

With these notations, the functional $F_{\varepsilon}$ becomes

$$
F_{\varepsilon}(u)=\int_{\mathbb{R}^{2}}\left(\tilde{f}_{1}-\mathcal{F}_{1} u\right)^{2} d x+\int_{\mathbb{R}^{2}}\left(\tilde{f}_{2}-\mathcal{F}_{2} u\right)^{2} d x+\int_{\Omega} \psi(|D u|), \quad u \in L^{2}(\Omega) .
$$

Fix $u \in B V(\Omega)$. Assume $\phi \in L^{2}(\Omega)$ belongs to $\partial F_{\varepsilon}(u)$, i.e. $u$ is the minimum on $B V(\Omega)$ of

$$
\inf _{v \in B V(\Omega)} F_{\varepsilon}(v)-\int_{\Omega} \phi v d x .
$$

The infimum on $B V(\Omega)$ of (9) is the same as the infimum on $W^{1,1}(\Omega)$ (see [35]), so we may replace $B V(\Omega)$ with $W^{1,1}(\Omega)$, and our primal problem $(\mathcal{P})$ is

$$
(\mathcal{P}) \quad \inf _{v \in W^{1,1}(\Omega)} F_{\varepsilon}(v)-\int_{\Omega} \phi v d x
$$

We now formulate the problem $\left(\mathcal{P}^{*}\right)$ dual to $(\mathcal{P})$ (see [15] for details on duality results applied here). The Legendre transform of a linear functional $\Phi: X \rightarrow \overline{\mathbb{R}}$ is $\Phi^{*}: X^{*} \rightarrow \overline{\mathbb{R}}$ given by

$$
\Phi^{*}\left(v^{*}\right)=\sup _{v \in X}\left\{\left\langle v, v^{*}\right\rangle-\Phi(v)\right\} .
$$

Let $\Lambda: W^{1,1}(\Omega) \rightarrow L^{2}\left(\mathbb{R}^{2}\right)^{2} \times L^{1}(\Omega)^{2}$ be the continuous linear operator $\Lambda v=$ $\left(\mathcal{F}_{1} v, \mathcal{F}_{2} v, D_{1} v, D_{2} v\right), \mathcal{F}: W^{1,1}(\Omega) \rightarrow \mathbb{R}, \mathcal{G}_{1}, \mathcal{G}_{2}: L^{2}\left(\mathbb{R}^{2}\right) \rightarrow \mathbb{R}, \mathcal{G}_{3}: L^{1}(\Omega)^{2} \rightarrow \mathbb{R}$ 
and $\mathcal{G}: L^{2}\left(\mathbb{R}^{2}\right)^{2} \times L^{1}(\Omega)^{2} \rightarrow \mathbb{R}$, such that

$$
\begin{aligned}
\mathcal{F}(v) & =-\int \phi v d x, \\
\mathcal{G}_{1}\left(w_{1}\right) & =\int_{\mathbb{R}^{2}}\left(\tilde{f}_{1}-w_{1}\right)^{2} d x, \quad \mathcal{G}_{2}\left(w_{2}\right)=\int_{\mathbb{R}^{2}}\left(\tilde{f}_{2}-w_{2}\right)^{2} d x, \\
\mathcal{G}_{3}(\bar{w}) & =\int_{\Omega} \psi(|\bar{w}|) d x, \\
\mathcal{G}(w) & =\mathcal{G}_{1}\left(w_{1}\right)+\mathcal{G}_{2}\left(w_{2}\right)+\mathcal{G}_{3}(\bar{w}), \quad w=\left(w_{1}, w_{2}, \bar{w}\right) \in L^{2}\left(\mathbb{R}^{2}\right)^{2} \times L^{1}(\Omega)^{2} .
\end{aligned}
$$

Then the problem $(\mathcal{P})$ can be written as:

$$
(\mathcal{P}) \inf _{v \in W^{1,1}(\Omega)}\{\mathcal{F}(v)+\mathcal{G}(\Lambda v)\} .
$$

Since $\mathcal{F}$ and $\mathcal{G}$ are proper (i.e. not identically $+\infty$ ) convex lower semi-continuous functions, the dual problem $\left(\mathcal{P}^{*}\right)[15]$ is

$$
\left(\mathcal{P}^{*}\right) \sup _{p^{*} \in L^{2}\left(\mathbb{R}^{2}\right)^{2} \times L^{\infty}(\Omega)^{2}}\left\{-\mathcal{F}^{*}\left(\Lambda^{*} p^{*}\right)-\mathcal{G}^{*}\left(-p^{*}\right)\right\},
$$

where

$$
\begin{aligned}
\mathcal{F}^{*}\left(\Lambda^{*} p^{*}\right) & :=\sup _{v \in W^{1,1}(\Omega)}\left\langle\Lambda^{*} p^{*}, v\right\rangle_{W^{1,1}(\Omega) \times\left(W^{1,1}(\Omega)\right)^{*}}+\int_{\Omega} \phi v d x \\
& =\sup _{v \in W^{1,1}(\Omega)}\left\langle\Lambda^{*} p^{*}+\phi, v\right\rangle_{W^{1,1}(\Omega) \times\left(W^{1,1}(\Omega)\right)^{*}} \\
& = \begin{cases}0, & \text { if } \Lambda^{*} p^{*}+\phi=0 \text { on } W^{1,1}(\Omega), \\
+\infty, & \text { otherwise. }\end{cases}
\end{aligned}
$$

It is easy to see $\mathcal{G}^{*}\left(p^{*}\right)=\mathcal{G}_{1}^{*}\left(p_{1}^{*}\right)+\mathcal{G}_{2}^{*}\left(p_{2}^{*}\right)+\mathcal{G}_{3}^{*}\left(\bar{p}^{*}\right)$, where $p^{*}=\left(p_{1}^{*}, p_{2}^{*}, \bar{p}^{*}\right) \in$ $L^{2}\left(\mathbb{R}^{2}\right)^{2} \times L^{\infty}(\Omega)^{2}$. Similarly $(i=1,2)$

$$
\mathcal{G}_{i}^{*}\left(p_{i}^{*}\right)=\int_{\mathbb{R}^{2}}\left(\frac{\left(p_{i}^{*}\right)^{2}}{4}+\tilde{f}_{i} p_{i}^{*}\right) d x, \quad \mathcal{G}_{3}^{*}\left(\bar{p}^{*}\right)= \begin{cases}\int_{\Omega} \psi^{*}\left(\left|\bar{p}^{*}\right|\right) d x, & \text { if }\left|\bar{p}^{*}\right| \leq \lambda, \\ +\infty, & \text { otherwise }\end{cases}
$$

Let $\mathcal{K}=\left\{p^{*} \in L^{2}\left(\mathbb{R}^{2}\right)^{2} \times L^{\infty}(\Omega)^{2}:\left|\bar{p}^{*}\right| \leq \lambda, \Lambda^{*} p^{*}+\phi=0\right.$ on $\left.W^{1,1}(\Omega)\right\}$, then $\left(\mathcal{P}^{*}\right)$ becomes

$$
\begin{aligned}
\left(\mathcal{P}^{*}\right) \sup _{p^{*} \in \mathcal{K}}\left\{E\left(p^{*}\right):=\right. & -\int_{\mathbb{R}^{2}}\left(\frac{\left(p_{1}^{*}\right)^{2}}{4}-\tilde{f}_{1} p_{1}^{*}\right) d x-\int_{\mathbb{R}^{2}}\left(\frac{\left(p_{2}^{*}\right)^{2}}{4}-\tilde{f}_{2} p_{2}^{*}\right) d x \\
& \left.-\int_{\Omega} \psi^{*}\left(\left|\bar{p}^{*}\right|\right) d x\right\} .
\end{aligned}
$$

The condition $\Lambda^{*} p^{*}+\phi=0$ on $W^{1,1}(\Omega)$ implies for any $w \in W^{1,1}(\Omega)$ 


$$
\begin{aligned}
0 & =\left\langle\Lambda^{*} p^{*}, w\right\rangle+\langle\phi, w\rangle=\left\langle p^{*}, \Lambda w\right\rangle+\langle\phi, w\rangle \\
& =\left\langle p_{1}^{*}, \mathcal{F}_{1} w\right\rangle+\left\langle p_{2}^{*}, \mathcal{F}_{2} w\right\rangle+\left\langle\bar{p}^{*}, D w\right\rangle+\langle\phi, w\rangle \\
& =\left\langle\mathcal{F}_{1}^{*} p_{1}^{*}+\mathcal{F}_{2}^{*} p_{2}^{*}-\operatorname{div}\left(\bar{p}^{*}\right)+\phi, w\right\rangle .
\end{aligned}
$$

Therefore $\mathcal{F}_{1}^{*} p_{1}^{*}+\mathcal{F}_{2}^{*} p_{2}^{*}-\operatorname{div}\left(\bar{p}^{*}\right)+\phi=0$ as a distribution in $\mathcal{D}^{\prime}(\Omega)$. Since $\mathcal{F}_{1}^{*} p_{1}^{*}, \mathcal{F}_{2}^{*} p_{2}^{*}$, and $\phi$ are in $L^{2}(\Omega)$, for $\bar{p}^{*}$ to satisfy this relation, we must have $\operatorname{div}\left(\bar{p}^{*}\right) \in L^{2}(\Omega)$. Therefore, following [4], we can define the trace $\bar{p}^{*} \cdot \vec{n}$ on $\Gamma=\partial \Omega$, where $\vec{n}$ is the outward unit normal to $\Gamma$. Moreover, applying integration by parts we obtain for all $v \in W^{1,1}(\Omega)$,

$$
\begin{aligned}
\int_{\Gamma}\left(\bar{p}^{*} \cdot \vec{n}\right) v d \Gamma= & \int_{\Omega} \sum_{j=1}^{2}\left(D_{j} \bar{p}_{j}^{*} v\right) d x+\int_{\Omega} \sum_{j=1}^{2}\left(\bar{p}_{j}^{*} D_{j} v\right) d x \\
= & \left\langle\mathcal{F}_{1}^{*} p_{1}^{*}, v\right\rangle+\left\langle\mathcal{F}_{2}^{*} p_{2}^{*}, v\right\rangle+\langle\phi, v\rangle \\
& -\left\langle p_{1}^{*}, \mathcal{F}_{1} v\right\rangle-\left\langle p_{2}^{*}, \mathcal{F}_{2} v\right\rangle-\langle v, \phi\rangle=0 .
\end{aligned}
$$

We deduce that if $p^{*}=\left(p_{1}^{*}, p_{2}^{*}, \bar{p}^{*}\right) \in \mathcal{K}$ then $\bar{p}^{*} \cdot \vec{n}=0 d \Gamma-$ a.e. on $\Gamma$. Thus

$$
\begin{aligned}
\mathcal{K}=\left\{p^{*}=\left(p_{1}^{*}, p_{2}^{*}, \bar{p}^{*}\right) \in L^{2}\left(\mathbb{R}^{2}\right)^{2} \times L^{\infty}(\Omega)^{2}:\right. \\
\\
\left.\quad\left|\bar{p}^{*}\right| \leq \lambda, \mathcal{F}_{1}^{*} p_{1}^{*}+\mathcal{F}_{2}^{*} p_{2}^{*}-\operatorname{div}\left(\bar{p}^{*}\right)+\phi=0 \text { in } \mathcal{D}(\Omega)^{*}, \bar{p}^{*} \cdot \vec{n}=0 \text { on } \Gamma\right\} .
\end{aligned}
$$

We have $\inf (\mathcal{P})<\infty$, the functional in $(\mathcal{P})$ is convex and continuous with respect to $\Lambda v$ in $L^{2}\left(\mathbb{R}^{2}\right)^{2} \times L^{1}(\Omega)^{2}$. Thus, by duality theorem [15, 28], $\left(\mathcal{P}^{*}\right)$ has a solution $M=\left(M_{1}, M_{2}, \bar{M}\right) \in \mathcal{K}$ and $\inf (\mathcal{P})=\sup \left(\mathcal{P}^{*}\right)$ (minimax relation), and we have

$$
F_{\varepsilon}(u)-\int_{\Omega} \phi u d x=E(M),
$$

or, since $M \in \mathcal{K}, \mathcal{F}_{1}^{*} M_{1}+\mathcal{F}_{2}^{*} M_{2}-\operatorname{div} \bar{M}+\phi=0$ in $\mathcal{D}^{\prime}(\Omega)$, (10) becomes

$$
\begin{aligned}
& \int_{\mathbb{R}^{2}}\left(\frac{M_{1}^{2}}{4}-\tilde{f}_{1} M_{1}\right) d x+\int_{\mathbb{R}^{2}}\left(\frac{M_{2}^{2}}{4}-\tilde{f}_{2} M_{2}\right) d x+\int_{\mathbb{R}^{2}}\left(\tilde{f}_{1}-\mathcal{F}_{1} u\right)^{2} d x \\
& \quad+\int_{\mathbb{R}^{2}}\left(\tilde{f}_{2}-\mathcal{F}_{2} u\right)^{2} d x+\int_{\Omega} \psi(|D u|)+\int_{\mathbb{R}^{2}} M_{1}\left(\mathcal{F}_{1} u\right) d x \\
& \quad+\int_{\mathbb{R}^{2}} M_{2}\left(\mathcal{F}_{2} u\right) d x-\int_{\Omega} u \operatorname{div} \bar{M} d x+\int_{\Omega} \psi^{*}(|\bar{M}|) d x=0 .
\end{aligned}
$$

Following [14, 35], we can associate to $u$ and $\bar{M}$ a bounded unsigned measure, denoted $D u \cdot \bar{M}$, defined as a distribution on $\Omega$ by $\langle D u \cdot \bar{M}, \varphi\rangle=-\int_{\Omega} u(\operatorname{div} \bar{M}) \varphi d x-$ $\int_{\Omega} \bar{M} \cdot(\nabla \varphi) u d x$, for all $\varphi \in \mathcal{D}(\Omega)$. By the generalized Green's formula (see also [4, 32]) $\int_{\Omega} D u \cdot \bar{M}=-\int_{\Omega} u \cdot \operatorname{div} \bar{M}+\int_{\Gamma} u(\bar{M} \cdot \vec{n}) d \Gamma$, and since $\bar{M} \cdot \vec{n}=0 \mathrm{~d} \Gamma$-a.e., (11) becomes

$$
\int_{\mathbb{R}^{2}}\left(\frac{M_{1}^{2}}{4}-\tilde{f}_{1} M_{1}\right) d x+\int_{\mathbb{R}^{2}}\left(\frac{M_{2}^{2}}{4}-\tilde{f}_{2} M_{2}\right) d x+\int_{\mathbb{R}^{2}}\left(\tilde{f}_{1}-\mathcal{F}_{1} u\right)^{2} d x
$$




$$
\begin{aligned}
& +\int_{\mathbb{R}^{2}}\left(\tilde{f}_{2}-\mathcal{F}_{2} u\right)^{2} d x+\int_{\Omega} \psi(|D u|)+\int_{\mathbb{R}^{2}} M_{1}\left(\mathcal{F}_{1} u\right) d x+\int_{\mathbb{R}^{2}} M_{2}\left(\mathcal{F}_{2} u\right) d x \\
& +\int_{\Omega} D u \cdot \bar{M}+\int_{\Omega} \psi^{*}(|\bar{M}|) d x=0 .
\end{aligned}
$$

Applying the decomposition $D u=\nabla u d x+C_{u}+\left.\left(u^{+}-u^{-}\right) \vec{n} d \mathcal{H}^{1}\right|_{S_{u}}$ (see [15, $17,35])$, where $C_{u}\left(S_{u}\right)=0, \nabla u d x$ is the Lebesgue part, $S_{u}$ is the jump part, and $C_{u}$ is the Cantor part, we have

$$
\begin{aligned}
& \int_{\mathbb{R}^{2}}\left(\frac{M_{1}^{2}}{4}-\tilde{f}_{1} M_{1}\right) d x+\int_{\mathbb{R}^{2}}\left(\frac{M_{2}^{2}}{4}-\tilde{f}_{2} M_{2}\right) d x+\int_{\mathbb{R}^{2}}\left(\tilde{f}_{1}-\mathcal{F}_{1} u\right)^{2} d x \\
& \quad+\int_{\mathbb{R}^{2}}\left(\tilde{f}_{2}-\mathcal{F}_{2} u\right)^{2} d x+\int_{\Omega}\left[\psi(|\nabla u|)+\nabla u \cdot \bar{M}+\psi^{*}(|\bar{M}|)\right] d x+\lambda \int_{\Omega \backslash S_{u}}\left|C_{u}\right| \\
& \quad+\lambda \int_{S_{u}}\left(u^{+}-u^{-}\right) d \mathcal{H}^{1}+\int_{\mathbb{R}^{2}} M_{1}\left(\mathcal{F}_{1} u\right) d x+\int_{\mathbb{R}^{2}} M_{2}\left(\mathcal{F}_{2} u\right) d x+\int_{\Omega \backslash S_{u}} \bar{M} \cdot C_{u} \\
& \quad+\int_{S_{u}}\left(u^{+}-u^{-}\right) \bar{M} \cdot \vec{n} d \mathcal{H}^{1}=0 .
\end{aligned}
$$

From the definitions of $\mathcal{G}_{1}^{*}\left(-M_{1}\right), \mathcal{G}_{2}^{*}\left(-M_{2}\right)$, and $\mathcal{G}_{3}^{*}(-\bar{M})$, we have

1. $\left(\frac{M_{1}^{2}}{4}-\tilde{f}_{1} M_{1}\right)+\left(\tilde{f}_{1}-\mathcal{F}_{1} u\right)^{2}+M_{1}\left(\mathcal{F}_{1} u\right) \geq 0$ for $d x$ a.e.

2. $\left(\frac{M_{2}^{2}}{4}-\tilde{f}_{2} M_{2}\right)+\left(\tilde{f}_{2}-\mathcal{F}_{2} u\right)^{2}+M_{2}\left(\mathcal{F}_{2} u\right) \geq 0$ for $d x$ a.e.

3. $\psi(|\nabla u|)+\nabla u \cdot \bar{M}+\psi^{*}(|\bar{M}|) \geq \psi(|\nabla u|)-|\nabla u||\bar{M}|+\psi^{*}(|\bar{M}|) \geq 0$, dx a.e. in $\Omega$.

By the Radon-Nikodym derivative theorem, we have $C_{u} \ll\left|C_{u}\right|$ and $\exists h \in$ $L^{1}\left(\left|C_{u}\right|\right)^{2}$ such that $|h|=1$ and $C_{u}=h \cdot\left|C_{u}\right|$. Thus, $\bar{M} \cdot C_{u}=\bar{M} \cdot h\left|C_{u}\right|$, hence

4. $\left(\bar{M} C_{u}+\lambda\left|C_{u}\right|\right)=(\lambda+\bar{M} \cdot h)\left|C_{u}\right| \geq 0$, since $|\bar{M}| \leq \lambda$.

Finally, when $u^{+}$and $u^{-}$are defined on $S_{u}$, we have $u^{+}-u^{-} \geq 0$ and $\lambda+\bar{M}$. $\vec{n} \geq 0$ (since $|\bar{M}| \leq \lambda$ ), hence

5. $\left(u^{+}-u^{-}\right)(\lambda+\bar{M} \cdot \vec{n}) \geq 0$ for $d \mathcal{H}^{1}$ a.e. in $S_{u}$.

In order for (12) to hold, we must have each of $1,2,3,4,5$ to be exactly 0 . Recall that $u$ is a minimizer of $F_{\varepsilon}$ if and only if $0 \in \partial F_{\varepsilon}(u)$. By setting the above $\phi$ to zero, we obtain a full characterization of the minimizer $u$ which can be stated as follows:

Theorem 4.1 Let $\mathcal{F}_{1}, \mathcal{F}_{2}, \tilde{f}_{1}, \tilde{f}_{2}$ be defined as above. Let $u \in B V(\Omega)$, with $D u=$ $\nabla u d x+C_{u}+J_{u}$ the decomposition of $D u$. Then $u$ is the minimizer of $F_{\varepsilon}$ if and only if there exists $M(x)=\left(M_{1}(x), M_{2}(x), \bar{M}(x)\right) \in L^{2}\left(\mathbb{R}^{2}\right) \times L^{2}\left(\mathbb{R}^{2}\right) \times L^{\infty}(\Omega)^{2}$, $|\bar{M}(\cdot)| \leq \lambda$, such that

$$
\begin{aligned}
& \psi(|\nabla u|)+\nabla u \cdot \bar{M}+\psi^{*}(|\bar{M}|)=0, \quad \text { dx a.e. } x \in \Omega, \\
& \bar{M} \cdot \vec{n}=0 \quad \text { d } \Gamma \text { a.e. on } \Gamma=\partial \Omega,
\end{aligned}
$$




$$
\begin{aligned}
& \mathcal{F}_{1}^{*} M_{1}+\mathcal{F}_{2}^{*} M_{2}-\operatorname{div} \bar{M}=0 \quad \text { in } \mathcal{D}^{\prime}(\Omega), \\
& -M_{1}=2\left(\mathcal{F}_{1} u-\tilde{f}_{1}\right), \quad-M_{2}=2\left(\mathcal{F}_{2} u-\tilde{f}_{2}\right) \quad \text { dx a.e. } x \in \Omega, \\
& \lambda+\bar{M} \cdot \vec{n}=0, \quad|\bar{M}|=\lambda \quad d \mathcal{H}^{1} \text { a.e. } x \in S_{u}, \\
& \operatorname{supp}\left(\left|C_{u}\right|\right) \subset \mathcal{N}=\left\{x \in \Omega \backslash S_{u}, \lambda+\bar{M}(x) \cdot h(x)=0,\right. \\
& \left.\quad h \in L^{1}\left(\left|C_{u}\right|\right)^{2},|h|=1, C_{u}=h\left|C_{u}\right|\right\} .
\end{aligned}
$$

If $\varepsilon>0(\psi$ differentiable $)$, we can compute $\bar{M}$ at Lebesgue points $x$ of $|D u|$, dx a.e. in $\Omega$, by

$$
\bar{M}(x)=-\lambda \frac{\nabla u(x)}{\sqrt{\varepsilon^{2}+|\nabla u(x)|^{2}}} .
$$

Assertions (13)-(18) follow directly from above, while assertion (19) can be shown as in [35].

\subsubsection{The Simplified Euler-Lagrange Equation}

In practice, we work with the regularized functional $F_{\varepsilon}, \varepsilon>0$ small, and we neglect the singular part $D_{s} u$ of $D u$, so we set $D u \approx \nabla u d x$. Thus the optimality conditions for $u$ and $\nabla u, d x-$ a.e. in $\Omega$, that we use in practice, are given by (14)-(16), together with (19) (remember we impose $u=0$ outside $\bar{\Omega}$ when $\hat{u}$ appears).

Recall the two identities $\int v \hat{w} d x=\int \hat{v} w d x$ and $\int v \overline{\hat{w}} d x=\int \overline{\hat{v}} w d x$, for any $w(x) \in \mathbb{R}$. Together with (14)-(16), (19), after some manipulations, we obtain the simplified Euler-Lagrange equation

$$
\begin{cases}\lambda \operatorname{div}\left(\frac{\nabla u}{\sqrt{\varepsilon^{2}+|\nabla u|^{2}}}\right)+K^{*}\left(2 \boldsymbol{R e}\left\{\mathfrak{F}\left(\frac{\overline{\hat{u}}_{0}-\overline{\widehat{K u}}}{\left(1+|\xi|^{2}\right)^{s}}\right)\right\}\right)=0, & \text { in } \Omega, \\ \frac{\nabla u}{\sqrt{\varepsilon^{2}+|\nabla u|^{2}}} \cdot \vec{n}=0, & \text { on } \partial \Omega, \\ u=0, & \text { outside } \bar{\Omega} .\end{cases}
$$

\section{Remark 4.1}

(i) When $s=0$ and $K$ is the identity operator, the Euler-Lagrange equation in (20) above is the same as that of the regularized TV model (because $u_{0}-u=$ $\mathfrak{F}\left(\overline{\hat{u_{0}}}-\overline{\hat{u}}\right)$ when $u_{0}$ and $u$ are real-valued). This proves that the obtained PDE is consistent with the regularized version of the energy (5), (see also Remark 2.1(i)).

(ii) When the operator $K$ is defined in the Fourier domain by a multiplier $\hat{k}$ with tempered distributions, i.e. $\widehat{K \varphi}=\hat{k} \hat{\varphi}$, (20) becomes

$$
\lambda \operatorname{div}\left(\frac{\nabla u}{\sqrt{\varepsilon^{2}+|\nabla u|^{2}}}\right)+2 \operatorname{Re}\left\{\mathfrak{F}\left(\frac{\overline{\hat{u}}_{0}-\overline{\hat{k}} \overline{\hat{u}}}{\left(1+|\xi|^{2}\right)^{s}} \hat{k}\right)\right\}=0 .
$$


In fact, $\mathfrak{F}\left(\frac{\overline{\hat{u}}_{0}-\overline{\hat{k}} \overline{\hat{u}}}{\left(1+|\xi|^{2}\right)^{s}} \hat{k}\right)$ is real-valued, hence we get

$$
\lambda \operatorname{div}\left(\frac{\nabla u}{\sqrt{\varepsilon^{2}+|\nabla u|^{2}}}\right)+2 \mathfrak{F}\left(\frac{\overline{\hat{u}}_{0}-\overline{\hat{k}} \overline{\hat{u}}}{\left(1+|\xi|^{2}\right)^{s}} \hat{k}\right)=0 .
$$

(iii) If we integrate both sides of (21) over $\mathbb{R}^{2}$, using the above boundary conditions and that $u=0$ outside of $\bar{\Omega}$, then the first term vanishes, and we get

$$
\int_{\mathbb{R}^{2}} \mathfrak{F}\left(\frac{\overline{\hat{u}}_{0}-\overline{\hat{k}} \overline{\hat{u}}}{\left(1+|\xi|^{2}\right)^{s}} \hat{k}\right)=0, \quad \text { or } \quad \mathfrak{F}\left(\mathfrak{F}\left(\frac{\overline{\hat{u}}_{0}-\overline{\hat{k}} \overline{\hat{u}}}{\left(1+|\xi|^{2}\right)^{s}} \hat{k}\right)\right)(0)=0 .
$$

If we write $w(\xi)=\frac{\hat{u}_{0}(\xi)-\hat{k}(\xi) \hat{u}(\xi)}{\left(1+|\xi|^{2}\right)^{s}} \overline{\hat{k}}(\xi)$, then $\bar{w}(\xi)=w(-\xi)$ and

$$
\hat{\bar{w}}(x)=\int_{\mathbb{R}^{2}} w(-\xi) e^{-2 \pi i \xi \cdot x} d \xi=\int_{\mathbb{R}^{2}} w(\xi) e^{2 \pi i \xi \cdot x} d \xi=\check{w}(x) .
$$

Therefore, $\hat{\tilde{w}}(0)=w(0)=\left(\hat{u}_{0}(0)-\hat{k}(0) \hat{u}(0)\right) \overline{\hat{k}}(0)=0$. We impose that $\hat{k}(0) \neq 0$, that is, $k$ has nonzero mean, then $\hat{u}_{0}(0)-\hat{u}(0)=0$, which means that the component $v=u_{0}-u$ has zero mean, as in the $\left(B V(\Omega), L^{2}(\Omega)\right)$ model.

\subsection{Characterization of Minimizers via "Texture" Norm}

In this section we present additional results that characterize the solution to our proposed minimization problem (5), introducing a dual "texture" norm. Related characterizations of minimizers of (1) can be found in [24] and [4]. We begin by defining a semi-norm $\|\cdot\|_{*}$ on $H^{-s}$ dual to the $|\cdot|_{B V}$ norm as follows:

$$
\|f\|_{*}=\sup _{u \in B V(\Omega),|u|_{B V(\Omega)} \neq 0} \frac{\left|\mathbf{R e}\langle f, u\rangle_{-s}\right|}{|u|_{B V(\Omega)}}, \quad f \in H^{-s} .
$$

$\|\cdot\|_{*}$ is a semi-norm since for any $f, g \in H^{-s}$ and $u \in B V(\Omega), \operatorname{Re}\langle f+g, u\rangle_{-s}=$ $\boldsymbol{\operatorname { R e }}\langle f, u\rangle_{-s}+\boldsymbol{\operatorname { R e }}\langle g, u\rangle_{-s}$, so $\left|\boldsymbol{R e}\langle f+g, u\rangle_{-s}\right| \leq\left|\mathbf{R e}\langle f, u\rangle_{-s}\right|+\left|\boldsymbol{R e}\langle g, u\rangle_{-s}\right|$. This implies the triangle inequality. Moreover, it is clear that for any $\lambda \in \mathbb{R},\|\lambda f\|_{*}=$ $|\lambda|\|f\|_{*}$.

Lemma 4.1 If $f \in H^{-s}$ is such that $\|f\|_{*}<\infty$, then $\operatorname{Re}\left\langle f, 1_{\Omega}\right\rangle_{-s}=0$.

Proof Let $u \in B V(\Omega)$ be such that $|u|_{B V(\Omega)} \neq 0$. Then for any constant $c \in \mathbb{R}$

$$
\frac{\left|\boldsymbol{R e}\langle f, u+c\rangle_{-s}\right|}{|u+c|_{B V(\Omega)}}=\frac{\left|\boldsymbol{R e}\langle f, u+c\rangle_{-s}\right|}{|u|_{B V(\Omega)}}=\frac{\left|\boldsymbol{\operatorname { R e }}\left(\langle f, u\rangle_{-s}+\langle f, c\rangle_{-s}\right)\right|}{|u|_{B V(\Omega)}} \leq\|f\|_{*}<\infty .
$$

This implies that there is a constant $C$ such that $\left|\operatorname{Re}\langle f, c\rangle_{-s}\right| \leq C<\infty$ for any $c \in \mathbb{R}$. Then $|c|\left|\boldsymbol{R e}\left\langle f, 1_{\Omega}\right\rangle_{-s}\right| \leq C<\infty$ for any $c \in \mathbb{R}$, therefore $\left|\boldsymbol{R e}\left\langle f, 1_{\Omega}\right\rangle_{-s}\right|$ must be zero. 
Remark 4.2 Lemma 4.1 says that if $\|f\|_{*}$ defined by (22) is finite, then $\left|\boldsymbol{R e}\langle f, u\rangle_{-s}\right| \leq$ $|u|_{B V(\Omega)}\|f\|_{*}$ for any $u \in B V(\Omega)$.

Theorem 4.2 For $\lambda>0$, let $u$ be the unique minimizer of (5) and $v=u_{0}-K u$. Then

(I) $\left\|K^{*} u_{0}\right\|_{*} \leq \frac{\lambda}{2}$ if and only if $u=0, v=u_{0}$.

(II) Suppose $\left\|K^{*} u_{0}\right\|_{*}>\frac{\lambda}{2}$. Then $u \in B V(\Omega), v=u_{0}-K u$ is minimizer if and only if $\left\|K^{*} v\right\|_{*} \leq \frac{\lambda}{2}$ and $\mathbf{R e}\left\langle K^{*} v, u\right\rangle_{-s}=\frac{\lambda}{2}|u|_{B V}$. If, in addition $|u|_{B V(\Omega)} \neq 0$, then $\left\|K^{*} v\right\|_{*}=\frac{\lambda}{2}$.

Proof (I) The minimizer is $u=0, v=u_{0}$ if and only if for any $h \in B V(\Omega)$, any $\varepsilon \in \mathbb{R}$, $\varepsilon \neq 0$,

$$
\lambda|\varepsilon h|_{B V(\Omega)}+\left\|u_{0}-\varepsilon K h\right\|_{-s}^{2} \geq\left\|u_{0}\right\|_{-s}^{2} .
$$

Expanding the term $\left\|u_{0}-\varepsilon K h\right\|_{-s}^{2}$, changing $\varepsilon$ into $-\varepsilon$, dividing both sides by $|\varepsilon|$ and letting $|\varepsilon| \rightarrow 0$, we obtain

$$
\lambda|h|_{B V(\Omega)} \geq\left|\mathbf{R e}\left\langle u_{0}, K h\right\rangle_{-s}\right|=\left|\operatorname{Re}\left\langle K^{*} u_{0}, h\right\rangle_{-s}\right|, \quad \text { for all } h \in B V(\Omega) .
$$

Hence, $\left\|K^{*} u_{0}\right\|_{*} \leq \frac{\lambda}{2}$.

Conversely, if $\left\|K^{*} u_{0}\right\|_{*} \leq \frac{\lambda}{2}$, then by Remark 4.2, the last inequality (23) holds for any $h \in B V(\Omega)$. Adding $\left\|u_{0}\right\|_{-s}^{2}$ to both sides of (23) and $\|K h\|_{-s}^{2}$ to the left hand side, we obtain

$$
\lambda|h|_{B V(\Omega)}+\left\|u_{0}\right\|_{-s}^{2}-2 \operatorname{Re}\left\langle u_{0}, K h\right\rangle_{-s}+\|K h\|_{-s}^{2} \geq\left\|u_{0}\right\|_{-s}^{2} .
$$

Thus $u=0$ is a minimizer because for any $h \in B V(\Omega), \lambda|h|_{B V(\Omega)}+\left\|u_{0}-K h\right\|_{-s}^{2} \geq$ $\left\|u_{0}\right\|_{-s}^{2}$.

(II) We have $u \in B V(\Omega), v=u_{0}-K u$ is minimizer if and only if for any $h \in$ $B V(\Omega)$, any $\varepsilon \in \mathbb{R}:$

$$
\lambda|u+\varepsilon h|_{B V(\Omega)}+\|v-\varepsilon K h\|_{-s}^{2} \geq \lambda|u|_{B V(\Omega)}+\|v\|_{-s}^{2} .
$$

Similarly, by triangle inequality for $|u+\varepsilon h|_{B V(\Omega)}$, expending the quadratic term on the left-hand side, changing $\varepsilon$ into $-\varepsilon$, dividing both sides by $|\varepsilon|$ and letting $\varepsilon \rightarrow 0$, we obtain for any $h \in B V(\Omega)$

$$
\frac{\lambda}{2}|h|_{B V(\Omega)} \geq\left|\mathbf{R e}\left\langle K^{*} v, h\right\rangle_{-s}\right| .
$$

Thus, $\left\|K^{*} v\right\|_{*} \leq \frac{\lambda}{2}$.

Letting $h=u$ and $-1<\varepsilon<0$ in (24), we get

$$
-\lambda|\varepsilon||u|_{B V(\Omega)}+2|\varepsilon| \mathbf{R e}\left\langle K^{*} v, u\right\rangle_{-s}+\varepsilon^{2}\|K u\|_{-s}^{2} \geq 0 .
$$

Dividing both sides by $|\varepsilon|$ and letting $\varepsilon \nearrow 0$, we obtain $2 \mathbf{R e}\left\langle K^{*} v, u\right\rangle_{-s} \geq \lambda|u|_{B V(\Omega)}$. 
Since $\left|\boldsymbol{R e}\left\langle K^{*} v, u\right\rangle_{-s}\right| \leq \frac{\lambda}{2}|u|_{B V(\Omega)}$, we get $\left|\boldsymbol{R e}\left\langle K^{*} v, u\right\rangle_{-s}\right|=\frac{\lambda}{2}|u|_{B V(\Omega)}$. If $|u|_{B V(\Omega)} \neq 0$, then $\left\|K^{*} v\right\|_{*}=\frac{\lambda}{2}$.

Conversely, suppose $u \in B V(\Omega)$ and $v=u_{0}-K u$ satisfy $\left\|K^{*} v\right\|_{*} \leq \frac{\lambda}{2}$ (with equality if $\left.|u|_{B V(\Omega)} \neq 0\right)$ and $\operatorname{Re}\left\langle K^{*} v, u\right\rangle_{-s}=\frac{\lambda}{2}|u|_{B V(\Omega)}$. Then for any $h \in B V(\Omega)$ and $\varepsilon \in \mathbb{R}$, we have

$$
\begin{aligned}
\lambda|u+\varepsilon h|_{B V(\Omega)}+\|v-\varepsilon K h\|_{-s}^{2} \geq & 2 \operatorname{Re}\left\langle K^{*} v, u+\varepsilon h\right\rangle_{-s}+\|v\|_{-s}^{2} \\
& -2 \varepsilon \mathbf{R e}\langle v, K h\rangle_{-s}+\varepsilon^{2}\|K h\|_{-s}^{2} \\
= & \|v\|_{-s}^{2}+2 \operatorname{Re}\left\langle K^{*} v, u\right\rangle_{-s}+\varepsilon^{2}\|K h\|_{-s}^{2} \\
\geq & \|v\|_{-s}^{2}+\lambda|u|_{B V(\Omega)} .
\end{aligned}
$$

Therefore, $u$ is a minimizer.

\section{Remark 4.3}

(i) Note that if $\mathbf{R e}\left\langle u_{0}, K 1_{\Omega}\right\rangle_{-s}=0$ (can be simply obtained by subtracting a constant from $u_{0}$ ), then in part (II) above we always have $|u|_{B V(\Omega)} \neq 0$ (because if $u$ is a constant minimizer, then $u$ must be zero in this case, but this cannot hold in part (II)).

(ii) The above characterization of minimizers holds if the total variation $|u|_{B V(\Omega)}$ is substituted by another functional $\Phi$ on $B V(\Omega)$ that is convex, lower semicontinuous and positive homogeneous of degree 1 .

\section{Numerical Approximation of the Model}

We consider the case of blurring operator $K$, defined by $\widehat{K u}=\hat{k} \hat{u}$. Applying gradient descent to our functional amounts to consider the time-dependent version of (21), $t \geq 0$, giving $u(0, x)$ in $\bar{\Omega}$,

$$
\begin{cases}\frac{\partial u}{\partial t}=\lambda \operatorname{div}\left(\frac{\nabla u}{\sqrt{\varepsilon^{2}+|\nabla u|^{2}}}\right)+2 \mathfrak{F}\left(\frac{\overline{\hat{u}}_{0}-\overline{\hat{k}} \overline{\hat{u}}}{\left(1+|\xi|^{2}\right)^{s}} \hat{k}\right), & \text { in } \Omega, \\ \frac{\nabla u}{\sqrt{\varepsilon^{2}+|\nabla u|^{2}}} \cdot \vec{n}=0, & \text { on } \partial \Omega, \\ u=0, & \text { outside } \bar{\Omega} .\end{cases}
$$

To proceed with the discretization of (25), let us assume that the initial discrete image $u_{0}$ is of $M \times M$ pixels, and that it has been sampled from its continuous version at uniformly spaced points starting at $(0,0)$, i.e. $u_{0, j, l}=u_{0}(j \triangle x, l \triangle x)$ for $j, l=0,1, \ldots, M-1$, where $\Delta x$ is to be determined. 


\subsection{The "Force" Term}

Computing the force term $\mathfrak{F}\left(\frac{\overline{\hat{u}}_{0}-\overline{\hat{k}} \overline{\hat{u}}}{\left(1+|\xi|^{2}\right)^{s}} \hat{k}\right)$ in (25) requires the Discrete Fourier Transform (DFT), which is defined for any $M \times M$ signal $u$ as

$$
\hat{u}_{m, n}=\frac{1}{M^{2}} \sum_{j, l=0}^{M-1} u_{j, l} e^{-2 \pi i(j m+l n) / M}, \quad \text { for } m, n=0,1, \ldots, M-1 .
$$

The Inverse Discrete Fourier Transform (IDFT) for $u$ is defined as

$$
\check{u}_{j, l}=\sum_{m, n=0}^{M-1} u_{m, n} e^{2 \pi i(j m+l n) / M}, \quad \text { for } j, l=0,1, \ldots, M-1 .
$$

The DFT array $(\hat{u})_{m, n}$ is, indeed, as taken from its continuous counterpart at frequencies $(m \triangle \xi, n \triangle \xi)(m, n=0,1, \ldots, M-1)$. The inverse relation between the DFT and IDFT implies that $\triangle x$ and $\triangle \xi$ are inversely related by

$$
\triangle \xi=\frac{1}{M \triangle x}
$$

Therefore, to give our numerical computations a balance weight between the spatial terms and the Fourier frequency terms, we shall choose

$$
\triangle x=\frac{1}{\sqrt{M}} \quad \text { and } \quad \Delta \xi=\frac{1}{\sqrt{M}} .
$$

A final note on computing the force term: before taking the DFT, we multiply $(-1)^{j+l}$ to the signal. This shifts the origin of the frequency domain to the center of the image. Thus, for $0 \leq m, n<M$, the $(m, n)$ entry corresponds to the Fourier coefficient at frequency $\left(\left(m-\frac{M}{2}\right) \triangle \xi,\left(n-\frac{M}{2}\right) \triangle \xi\right)$. Therefore, we evaluate the weight function $\frac{1}{\left(1+|\xi|^{2}\right)^{s}}$ at points $\xi_{1}, \xi_{2}=-\frac{M}{2} \triangle \xi,-\frac{(M-1)}{2} \triangle \xi, \ldots, \frac{(M-1)}{2} \triangle \xi$.

\subsection{The Curvature Term}

For the discrete gradient, we shall use the following usual notations:

$$
\begin{aligned}
\nabla^{+,+} u & =\left(\nabla_{x}^{+} u, \nabla_{y}^{+} u\right), & \nabla^{+,-} u & =\left(\nabla_{x}^{+} u, \nabla_{y}^{-} u\right), \\
\nabla^{-,+} u & =\left(\nabla_{x}^{-} u, \nabla_{y}^{+} u\right), & \nabla^{-,-} u & =\left(\nabla_{x}^{-} u, \nabla_{y}^{-} u\right),
\end{aligned}
$$

where

$$
\begin{aligned}
\nabla_{x}^{+} u & =u_{j+1, l}-u_{j, l}, & \nabla_{x}^{-} & =u_{j, l}-u_{j-1, l}, \\
\nabla_{y}^{+} u & =u_{j, l+1}-u_{j, l}, & \nabla_{y}^{-} & =u_{j, l}-u_{j, l-1} .
\end{aligned}
$$


Since the dual operators to $\nabla^{+,+}, \nabla^{+,-}, \nabla^{-,+}, \nabla^{-,-}$are, respectively, the operators $\operatorname{div}^{-,-}, \operatorname{div}^{-,+}, \operatorname{div}^{+,-}, \operatorname{div}^{+,+}$, we can write the regularized curvature term in one of four ways:

$$
\operatorname{div}\left(\frac{\nabla u}{\sqrt{\varepsilon^{2}+|\nabla u|^{2}}}\right) \approx \operatorname{div}^{\alpha *, \beta *}\left(\frac{\nabla^{\alpha, \beta} u}{\sqrt{\varepsilon^{2}+\left|\nabla^{\alpha, \beta} u\right|^{2}}}\right)
$$

where $\operatorname{div}^{\alpha *, \beta *}$ denotes the dual operator of $\nabla^{\alpha, \beta}$, with $\alpha, \beta=+,-$.

To make our numerical scheme rotationally invariant, we use all four approximations to the gradient operator by alternating them with each iteration [4]. For example, if $u^{n}$ was computed using $\nabla^{+,+}$, then we use $\nabla^{+,-}$to compute $u^{n+1}, \nabla^{-,+}$to compute $u^{n+2}$, and $\nabla^{-,-}$to compute $u^{n+3}$, and then repeat.

\subsection{Numerical Algorithm}

We solve (25) with the following iterative semi-implicit scheme [6, 35]:

1. $u^{0}$ is arbitrarily given (we can take $u^{0}=u_{0}$ )

2. Once $u^{n}$ is calculated, compute the forcing term $F^{n}=\mathfrak{F}\left(\frac{\overline{\hat{u}}_{0}-\overline{\hat{k}} \overline{\hat{u}}^{n}}{\left(1+|\xi|^{2}\right)^{s}} \hat{k}\right)$

3. Compute $u_{j, l}^{n+1}$, for $j, l=1,2, \ldots, M-2$ as the solution of the linear discrete equation: $u_{j, l}^{n+1}=u_{j, l}^{n}+\triangle t\left(\frac{\lambda}{\triangle x} \operatorname{div}^{\alpha *, \beta *}\left(\frac{\nabla^{\alpha, \beta} u^{n+1}}{\sqrt{\varepsilon^{2}+\left|\nabla^{\alpha, \beta} u^{n}\right|^{2}}}\right)_{j, l}+2 F_{j, l}^{n}\right)$ with $\varepsilon=\varepsilon^{\prime} \triangle x$, some $\varepsilon^{\prime}>0$ small, and the discrete boundary conditions $u_{0, l}^{n+1}=u_{1, l}^{n+1}, u_{M-1, l}^{n+1}=$ $u_{M-2, l}^{n+1}, u_{j, 0}^{n+1}=u_{j, 1}^{n+1}, u_{j, M-1}^{n+1}=u_{j, M-2}^{n+1}$ (since the denominator $\sqrt{\varepsilon^{2}+|\nabla u|^{2}}{ }_{j, l}$ in the discrete boundary condition is always strictly positive).

To clarify the notations in step 3 of our algorithm, assume that we are solving at pixel $(j, l)$ the equation

$$
u_{j, l}^{n+1}=u_{j, l}^{n}+\Delta t\left(\frac{\lambda}{(\triangle x)} \operatorname{div}^{-,-}\left(\frac{\nabla^{+,+} u^{n+1}}{\sqrt{\varepsilon^{2}+\left|\nabla^{+,+} u^{n}\right|^{2}}}\right)_{j, l}+2 F_{j, l}^{n}\right) .
$$

Let $d_{j, l}=\left(\sqrt{\varepsilon^{2}+\left|\nabla^{+,+} u^{n}\right|^{2}}\right)_{j, l}$, which is known since $u^{n}$ is already computed. Then let

$$
\begin{aligned}
\operatorname{div}^{-,-}\left(\frac{\nabla^{+,+} u^{n+1}}{\sqrt{\varepsilon^{2}+\left|\nabla^{+,+} u^{n}\right|^{2}}}\right)_{j, l}= & \frac{u_{j+1, l}^{n}-u_{j, l}^{n+1}}{d_{j, l}}-\frac{u_{j, l}^{n+1}-u_{j-1, l}^{n}}{d_{j-1, l}} \\
& +\frac{u_{j, l+1}^{n}-u_{j, l}^{n+1}}{d_{j, l}}-\frac{u_{j, l}^{n+1}-u_{j, l-1}^{n}}{d_{j, l-1}} .
\end{aligned}
$$

Basically, we set all terms at the current pixel $(j, l)$ in the curvature term to be unknown. Setting $c_{j, l}=\frac{\lambda \Delta t}{d_{j, l}(\Delta x)}$, we then have

$$
\begin{aligned}
\left(1+c_{j-1, l}+2 c_{j, l}+c_{j, l-1}\right) u_{j, l}^{n+1}= & u_{j, l}^{n}+c_{j-1, l} u_{j-1, l}^{n}+c_{j, l} u_{j+1, l}^{n} \\
& +c_{j, l} u_{j, l+1}^{n}+c_{j, l-1} u_{j, l-1}^{n}+2 \triangle t F_{j, l}^{n} .
\end{aligned}
$$


Hence, $u_{j, l}^{n+1}$ is obtained by dividing the coefficient $\left(1+c_{j-1, l}+2 c_{j, l}+c_{j, l-1}\right)$ to both sides of the equation. We remark here that the semi-implicit scheme above approaches the steady state equation much faster than an explicit scheme, and thus, an advantage.

\subsection{The Blurring Operator $K$}

In our implementation, we perform blurring directly in the frequency domain. To blur an image, we multiply pixel-wise a Gaussian kernel $\hat{k}$ to the DFT of the image, then we take the inverse transform. For a Gaussian kernel we used the form $\hat{k}\left(\xi_{1}, \xi_{2}\right)=\exp \left(-\frac{\xi_{1}^{2}+\xi_{2}^{2}}{2 / \alpha^{2}}\right)$. Thus, in our numerical computation, we never compute a discrete convolution, which is computationally more expensive.

\section{Numerical Results for Image Restoration}

In this section we present numerical results obtained by applying our proposed new model to image denoising, deblurring and decomposition. For comparison, we also present results from application of the ROF model [31] to the same images. In our implementation of both models (our proposed model and the ROF model), we discretize the curvature term in the manner given in Sect. 5.2. In our proposed model, $s$ is a parameter. We will show numerical results obtained with various values of $s$. For image restoration (denoising or deblurring), the parameter $\lambda$ was chosen so that the best residual-mean-squared-error $(R M S E)$ is obtained for each method. For the $R M S E$ and $S N R$, we use

$$
R M S E=\frac{\sqrt{\sum\left(u_{j, l}-u_{c, j, l}\right)^{2}}}{M N}, \quad S N R=\frac{\sum\left(u_{j, l}-\frac{\sum u_{j, l}}{M N}\right)^{2}}{\sum\left(u_{j, l}-u_{c, j, l}-\frac{\sum\left(u_{j, l}-u_{c, j, l}\right)}{M N}\right)^{2}},
$$

where $u_{c}$ is the clean image (which is known in all of our experiments), and $(M, N)$ is the size of the image.

We mention that alternative approaches for selecting $\lambda$ is the method proposed by Gilboa-Sochen-Zeevi [20], where the authors choose $\lambda$ in an optimal way, by maximizing an estimate of the $S N R$ or by minimizing the correlation between $u$ and $f-u$.

In Fig. 2, we show the denoising results obtained from our proposed new model with $H^{-1}$ and $H^{-0}$ norms in the fidelity and the ROF model performed on a synthetic piecewise constant image with additive Gaussian white noise of $\sigma=30$ (shown in Fig. 1). In Fig. 3, we show more results on the same image from our proposed model using $s=0.5$ and $s=2$, respectively, for the $H^{-s}$ norm in the fidelity term. We also show the results from our model using $H_{0}^{-1}$ semi-norm for the fidelity term (as discussed in Remark 2.1, equivalent case with the model from [27]). The RMSE and $S N R$, together with the value of the parameter $\lambda$, for all results in this experiment are shown in Table 1. Comparing the results, our model with $H^{-0.5}$ norm gives results with the best $R M S E$, while the $H_{0}^{-1}$ semi-norm gives results with the best SNR. Visually, the results from our model with $H^{-1}$ norm preserve best the edges in the $u$ 
Fig. 1 A synthetic image and its noisy version with additive Gaussian white noise with standard deviation 30 and zero mean
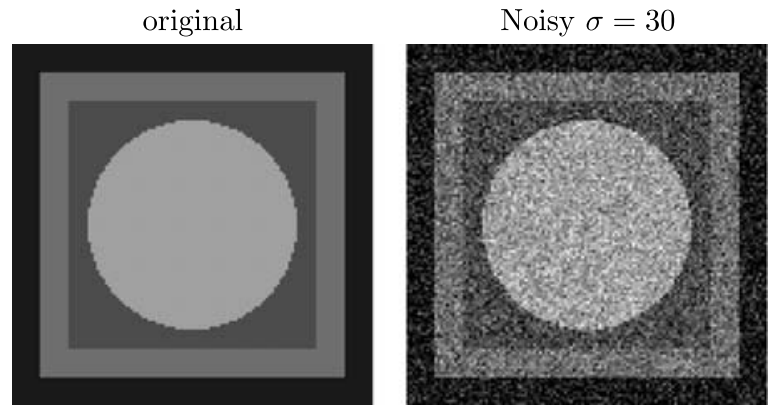

$u_{R O F}$

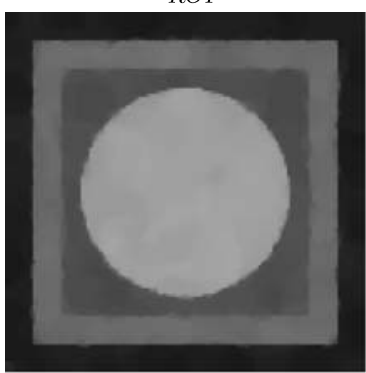

$v_{R O F}+60$

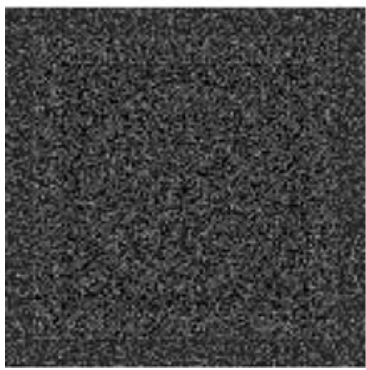

$u_{H^{-1}}$

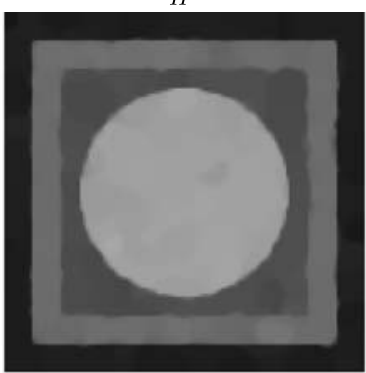

$v_{H^{-1}}+60$

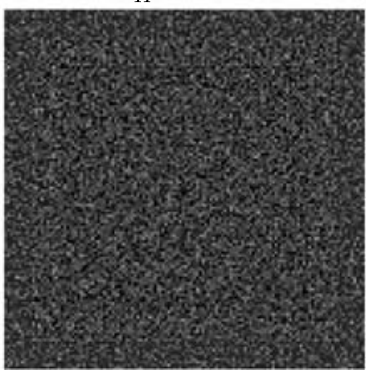

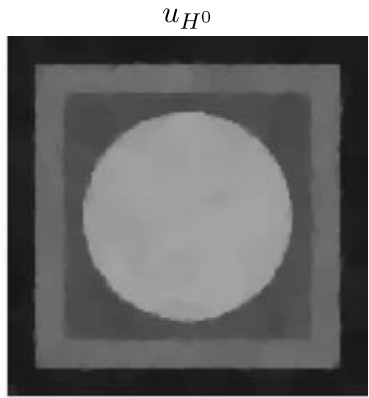

$v_{H^{0}}+60$

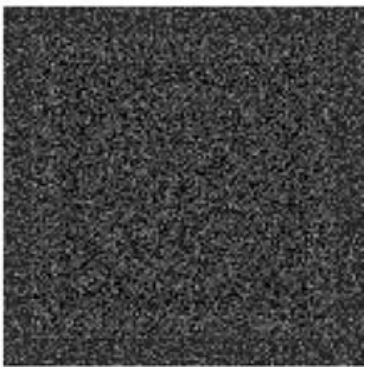

Fig. 2 Comparison of results from the ROF model with our proposed model $(s=0$ and $s=-1)$. Top: denoising results $u$ obtained from the ROF model, $H^{-1}$ and $H^{0}$. Bottom: corresponding residuals $v=u_{0}-u$. The case with $H^{0}$ is an equivalent calculation with ROF

Table 1 RMSE and SNR for the denoising results on the synthetic piecewise constant image shown in Figs. 2 and 3

\begin{tabular}{lclr}
\hline Restoration Model & $\lambda$ & $R M S E$ & \multicolumn{1}{l}{$S N R$} \\
\hline Noisy image & & 0.224578 & 4.03064 \\
ROF & 55 & 0.0526239 & 51.99021 \\
$H^{0}$ & 9.3 & 0.05204916 & 53.11084 \\
$H^{-0.5}$ & 3 & 0.04953237 & 58.99322 \\
$H^{-1}$ & 1.75 & 0.04959289 & 59.41552 \\
$H^{-2}$ & 0.9 & 0.05290355 & 52.67199 \\
$H_{0}^{-1}$ & 3.8 & 0.04977601 & 59.905 \\
\hline
\end{tabular}




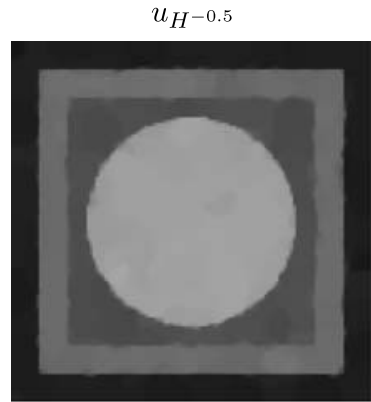

$v_{H^{-0.5}}+60$
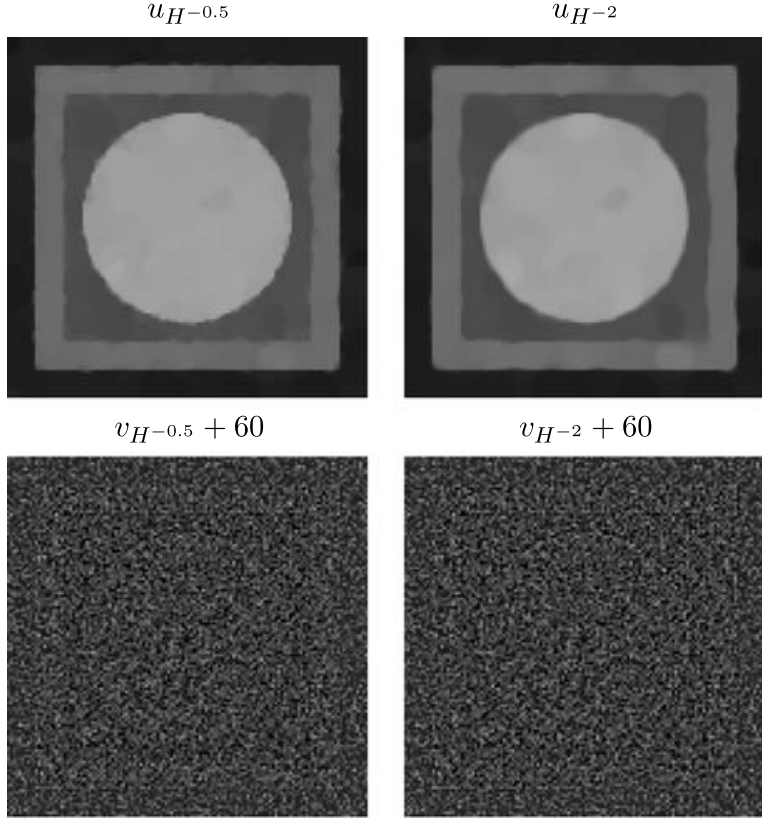

$v_{H^{-2}}+60$
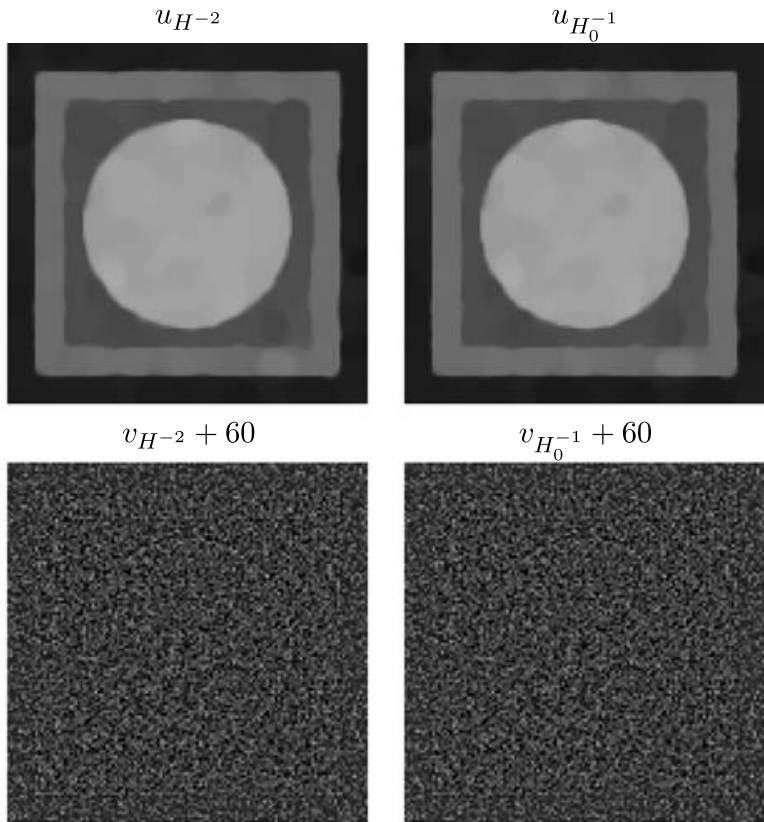

$v_{H_{0}^{-1}}+60$

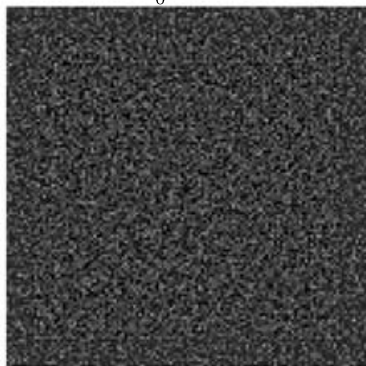

Fig. 3 More results from our proposed model, using for the norm in the fidelity term $H^{-0.5}$ (left), $H^{-2}$ (middle), and $H_{0}^{-1}$ semi-norm (right)

original

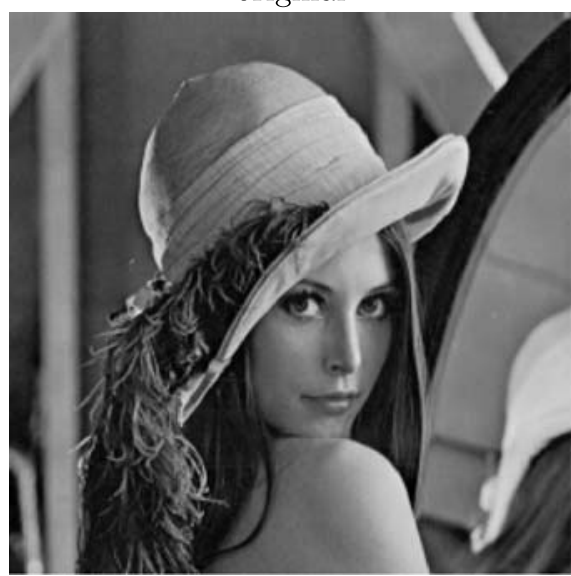

Noisy $\sigma=20$

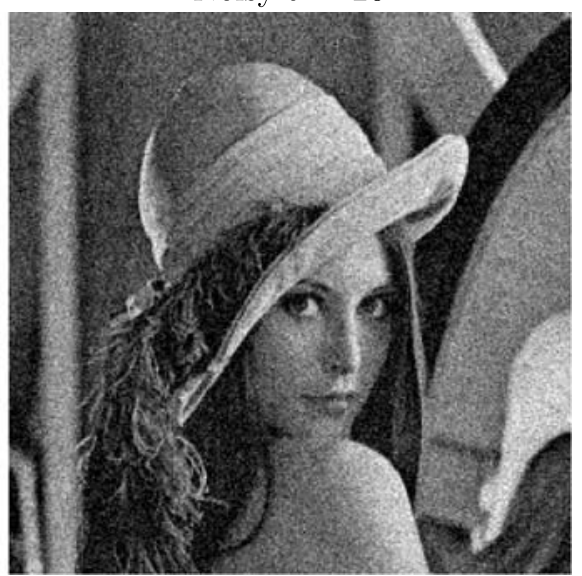

Fig. 4 Lena image and its noisy version with additive white noise

component. Overall, our proposed model performs much better than the ROF model, as expected.

In Fig. 5 we show results from another denoising application. We applied our model using $H^{-0.5}$ and $H^{-1}$ and the ROF model to a noisy image of a woman. The noise is additive white noise with standard deviation $\sigma=20$ (see Fig. 4). The RMSE 

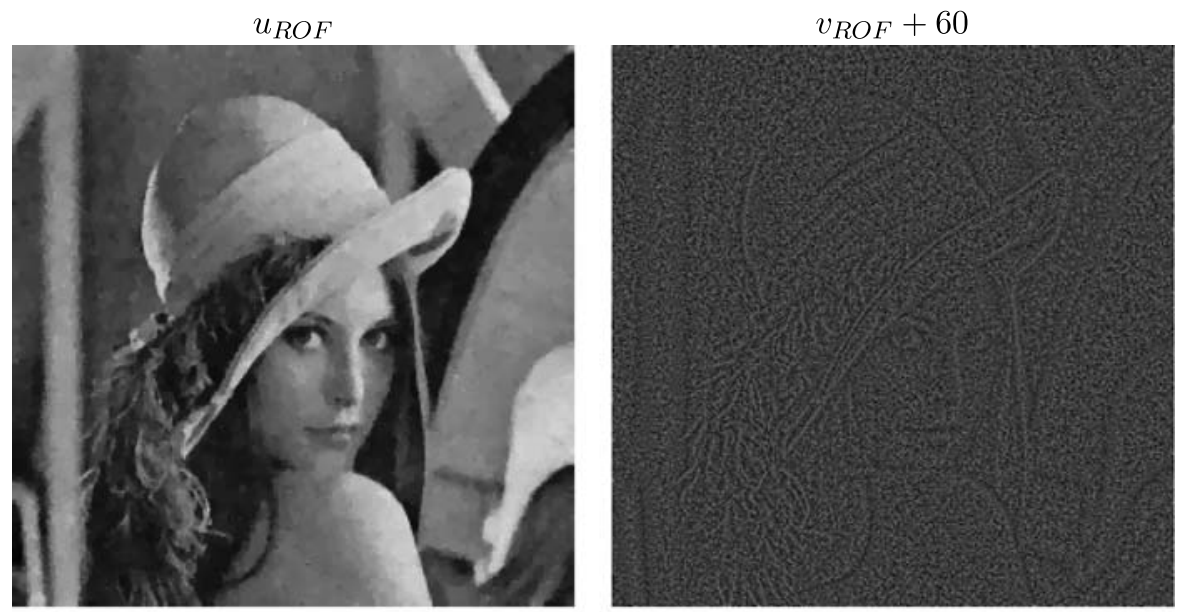

$u_{H^{-0.5}}$

$v_{H^{-0.5}}+60$
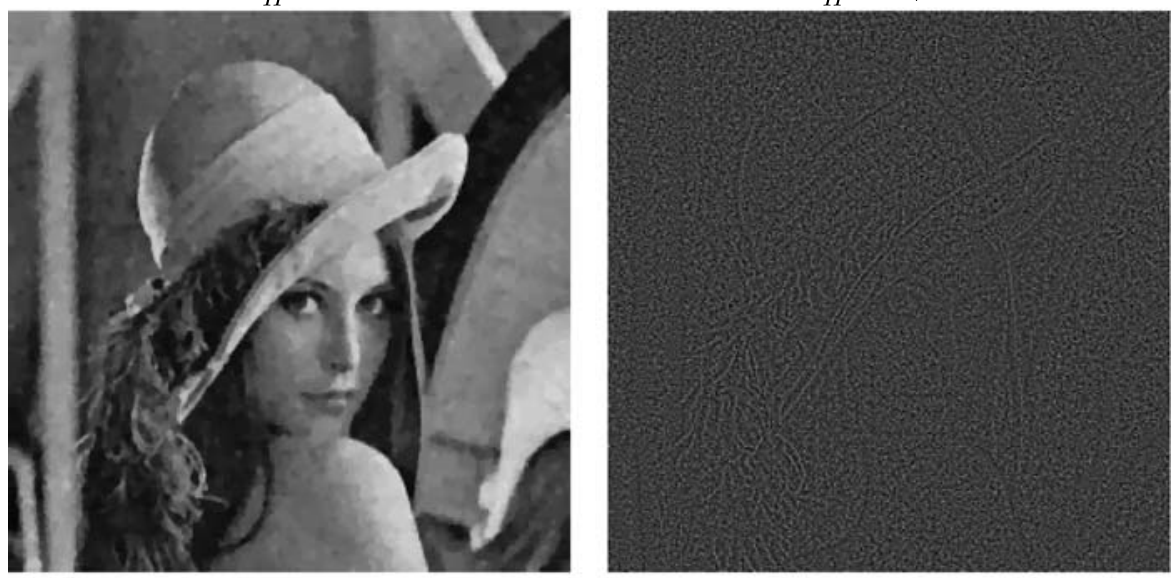

$u_{H^{-1}}$

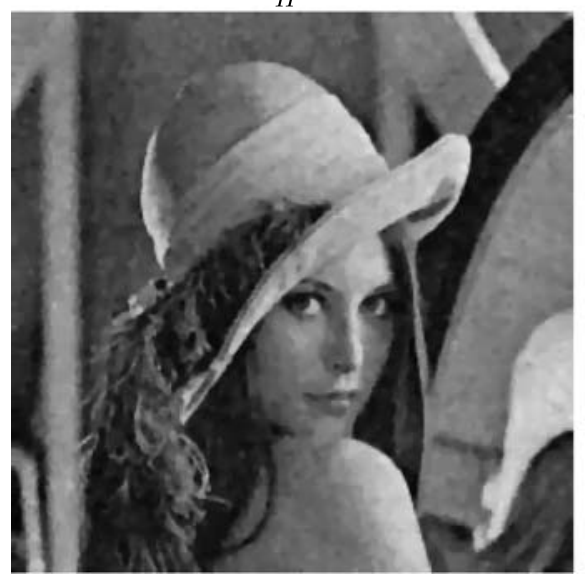

$v_{H^{-1}}+60$

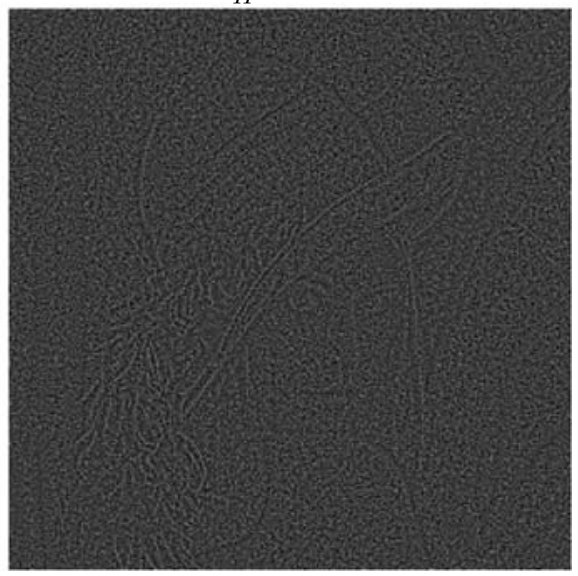

Fig. 5 Denoising results on Lena image. Result of ROF model (top), result of our model with $H^{-0.5}$ (middle), result of our model with $\mathrm{H}^{-1}$ (bottom) 
Fig. 6 Decomposition of a synthetic textured image. Results from ROF model $(\lambda=42)$ and our model with $H^{-1}(\lambda=0.5)$

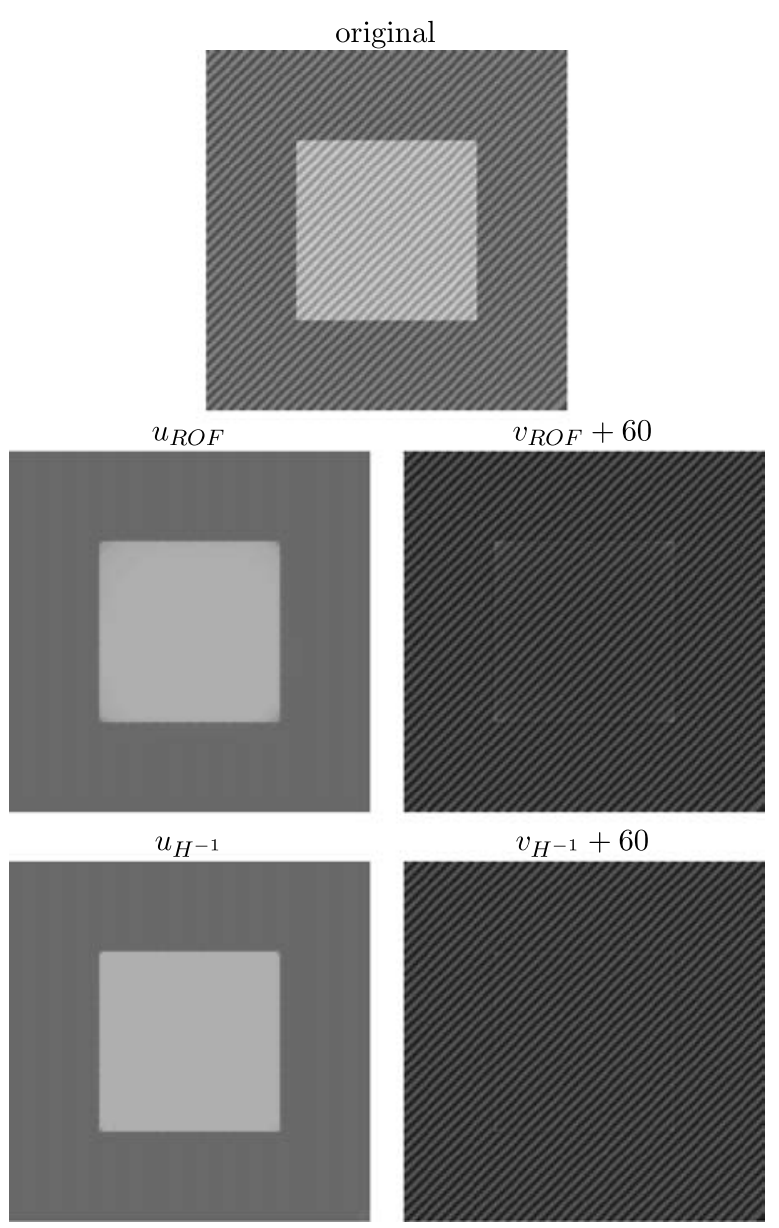

and $S N R$ of the original noisy image is 0.0762599 and 8.01743 , respectively. After denoising, the best results with ROF, $H^{-0.5}$, and $H^{-1}$ have the SNR of 30.64769, $32.97592,32.82501$, and the RMSE of $0.03548729,0.03461537,0.03472017$, respectively. The values for $\lambda$ that yield these results are $14,0.4,0.25$ for the ROF, $H^{-0.5}$, $H^{-1}$ model, respectively. Again, our model yields better results than the ROF model.

We try next texture removal with our $H^{-1}$-model and compare with ROF model. The results are shown in Figs. 6, 7. In Fig. 6, the texture image is synthetically created, and in Fig. 7, the image is natural. We can see that the $H^{-1}$-norm models better the texture than the $L^{2}$-norm.

In Figs. 8, 9, we show deblurring results on a synthetic image and a natural image of an office. The artificial blur is defined as described in Sect. 5.4 with $\alpha=0.8$. For the result in Fig. 8, the blurred image has $R M S E=0.1016$. The improved image has $R M S E=0.03618513$ using the parameter $\lambda=0.0011$. For the result in Fig. 9, the blurred image has $R M S E=0.181955$, and the improved image has $R M S E=0.10373$ using $\lambda=0.0004$. Visually, we see a significant improvement in the recovered image as compared to the degraded one. 
Fig. 7 Decomposition of a natural textured image. Results from ROF model $(\lambda=58$, middle) and our model with $H^{-1}(\lambda=2.5$, bottom $)$

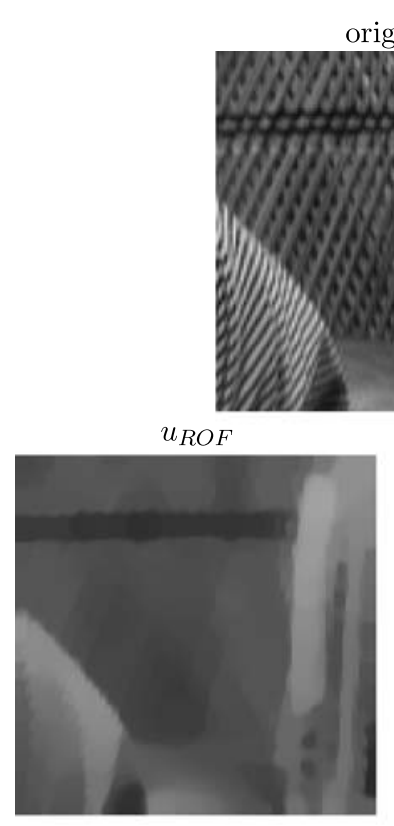

$u_{H^{-1}}$
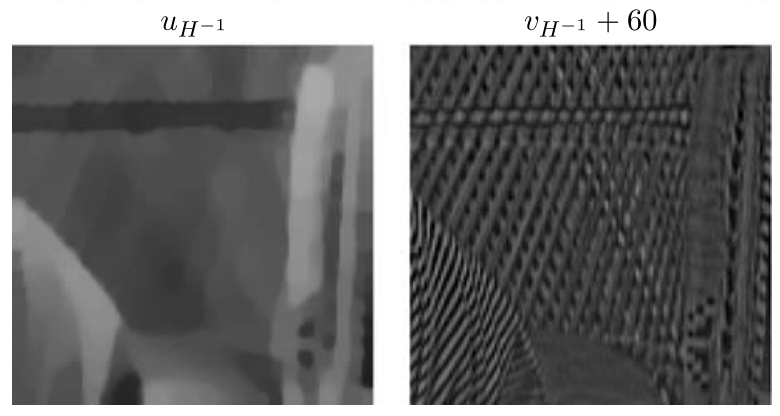

$v_{R O F}+60$

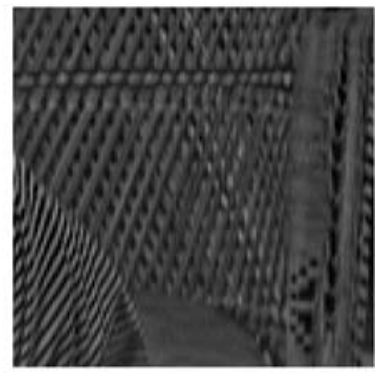

$v_{H^{-1}}+60$

blurred $(\alpha=0.8)$
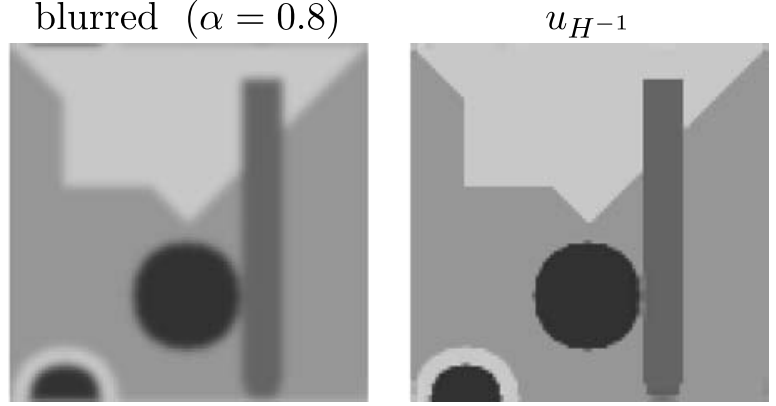

Fig. 8 Deblurring on a synthetic image. Result is obtained from our model with $H^{-1}(\lambda=0.0011)$ 

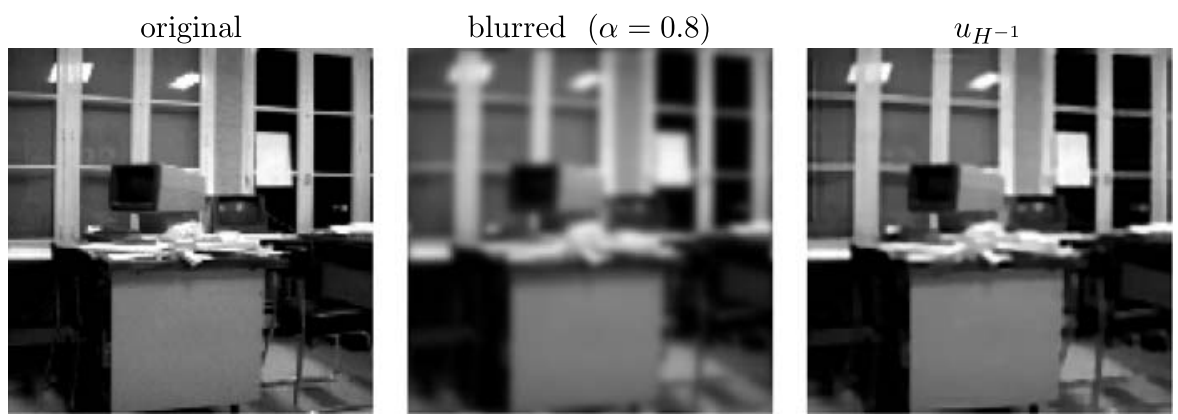

Fig. 9 Deblurring on image of an office. Result is obtained from our model with $H^{-1}(\lambda=0.0004)$

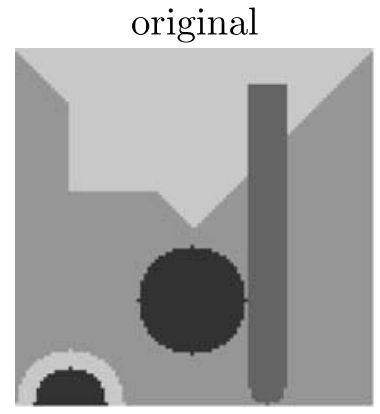

$u_{H^{-1}}$

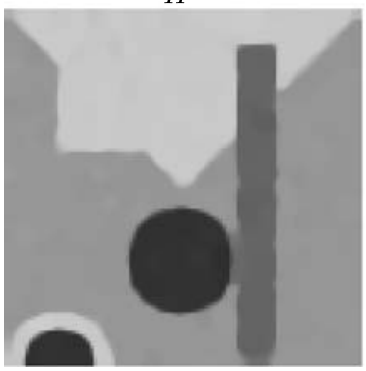

noisy blurred $(\alpha=0.8, \sigma=10)$

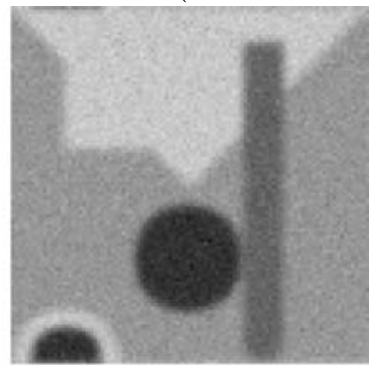

$v_{H^{-1}}+60$

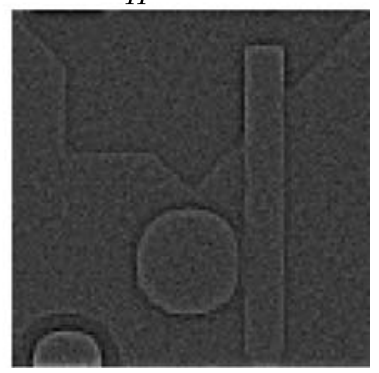

Fig. 10 Denoising-deblurring result using our model with $H^{-1}(\lambda=0.0675)$

Our last experiment is recovering from a blurred and noisy image. In Fig. 10, we add white noise of standard deviation $\sigma=10$ to the blurry image in Fig. 8. The degradation has $R M S E=0.121289$. The improved image is obtained with $\lambda=0.0675$, and the $R M S E=0.0607262$.

\section{Conclusion}

We have proposed a new variational model for image restoration and decomposition, using the Hilbert-Sobolev spaces of negative degree of differentiability to capture 
oscillatory patterns. We have presented an algorithm to solve the proposed variational problem. We have also presented results of numerical experiments for our proposed model and for the classical ROF model. In each experiment, we have chosen the parameter $\lambda$ so that the RMSE measurement is minimized. The results obtained from our proposed model are improved, visually and quantitatively (in terms of RMSE and SNR), over the ROF model, for values of $s \in[0.5,1]$. Outside this interval, we have obtained results similar with those produced by the ROF model.

In each of our numerical experiments, we choose manually the value for $s$. The results from our numerical experiments with synthetic and real images suggest that Gaussian white noise is captured best by the $H^{-s}$ norm when $0.5 \leq s \leq 1$. This agrees with Mumford and Gidas [25] whose work has motivated us to investigate the spaces $H^{-s}$ for modeling oscillatory patterns.

We also conclude that the value $s=1$ corresponding to $H^{-1}$ can be used both for image denoising as well as for cartoon and texture separation. However, we do not focus in this paper on $u+v+w$ decompositions, with $u$ a cartoon component, $v$ a texture component and $w$ a noise component, and we leave the study of separating noise from texture in a future work; we refer the reader to Aujol-Chambolle [9] for a solution in this direction.

Acknowledgements This work has been supported in part by an Alfred P. Sloan Fellowship, by the National Science Foundation (Grants NSF DMS 0312222 and ITR/AP 0113439) and by the National Institutes of Health through the NIH Roadmap for Medical Research, Grant U54 RR021813 entitled Center for Computational Biology (CCB). Finally, the authors would like to thank the anonymous referees for their useful remarks and suggestions.

\section{References}

1. Acar, R., Vogel, C.R.: Analysis of bounded variation penalty methods of ill-posed problems. Inverse Probl. 10, 1217-1229 (1994)

2. Alvarez, L., Gousseau, Y., Morel, J.M.: Scales in natural images and a consequence on their bounded variation norm. In: Lecture Notes in Computer Science, vol. 1682, pp. 247-258. Springer, London (1999)

3. Andreu-Vaillo, F., Ballester, C., Caselles, V., Mazon, J.M.: Minimizing total variation flow. C. R. Acad. Sci. Paris Sér. I Math. 331, 867-872 (2000)

4. Andreu-Vaillo, F., Caselles, V., Mazon, J.M.: Parabolic Quasilinear Equations Minimizing Linear Growth Functionals. Birkhauser, Basel (2004)

5. Aubert, G., Aujol, J.-F.: Modeling very oscillating signals. Application to image processing. AMO 51(2), 163-182 (2005)

6. Aubert, G., Vese, L.: A variational method in image recovery. SIAM J. Numer. Anal. 34(5), 19481979 (1997)

7. Aujol, J.-F., Aubert, G., Blanc-Féraud, L., Chambolle, A.: Image decomposition application to SAR images. In: Lecture Notes in Computer Science, vol. 2695, pp. 297-312. Springer, London (2003)

8. Aujol, J.-F., Aubert, G., Blanc-Féraud, L., Chambolle, A.: Image decomposition into a bounded variation component and an oscillating component. J. Math. Imaging Vis. 22(1), 71-88 (2005)

9. Aujol, J.-F., Chambolle, A.: Dual norms and image decomposition models. Int. J. Comput. Vis. 63(1), 85-104 (2005)

10. Chambolle, A., Lions, P.L.: Image recovery via total variation minimization and related problems. Numer. Math. 76, 167-188 (1997)

11. Daubechies, I., Teschke, G.: Wavelet-based image decompositions by variational functionals. In: Truchetet, F. (ed.) Wavelet Applications in Industrial Processing. Proc. SPIE, vol. 5266, pp. 94-105 (2004) 
12. Daubechies, I., Teschke, G.: Variational image restoration by means of wavelets: simultaneous decomposition, deblurring and denoising. Appl. Comput. Harmon. Anal. 19(1), 1-16 (2005)

13. Dautray, R., Lions, J.L.: Mathematical Analysis and Numerical Methods for Science and Technology, vol. 2. Springer, Berlin (1988)

14. Demengel, F., Temam, R.: Convex functions of a measure and applications. Indiana Univ. Math. J. 33(5), 673-709 (1984)

15. Ekeland, I., Temam, R.: Convex Analysis and Variational Problems. Am. Elsevier, New York (1976)

16. Esedoglu, S., Osher, S.J.: Decomposition of images by the anisotropic Rudin-Osher-Fatemi model. Commun. Pure Appl. Math. 57(12), 1609-1626 (2004)

17. Evans, L.C., Gariepy, R.F.: Measure Theory and Fine Properties of Functions. CRC Press, Boca Raton (1992)

18. Garnett, J.B., Le, T.M., Meyer, Y., Vese, L.A.: Image decompositions using bounded variation and homogeneous Besov spaces. Appl. Comput. Harmon. Anal. 23, 25-56 (2007)

19. Geman, S., Geman, D.: Stochastic relaxation, Gibbs distributions, and the Bayesian restoration of images. IEEE Trans. Pattern Anal. Mach. Intell. 6(6), 721-741 (1984)

20. Gilboa, G., Sochen, N., Zeevi, Y.Y.: Estimation of the optimal variational parameter via SNR analysis. In: Lecture Notes in Computer Science, vol. 3459, pp. 230-241. Springer, London (2005)

21. Gousseau, Y., Morel, J.M.: Are natural images of bounded variation? SIAM J. Math. Anal. 33(3), 634-648 (2001)

22. Le, T., Vese, L.A.: Image decomposition using total variation and $\operatorname{div}(\mathrm{BMO})$. Multiscale Model. Simul. 4(2), 390-423 (2005)

23. Levine, S.: An adaptive variational model for image decomposition. In: Lecture Notes in Computer Science, vol. 3757, pp. 382-397. Springer, London (2005)

24. Meyer, Y.: Oscillating Patterns in Image Processing and Nonlinear Evolution Equations. Univ. Lecture Ser., vol. 22. Am. Math. Soc., Providence (2002)

25. Mumford, D., Gidas, B.: Stochastic models for generic images. Q. Appl. Math. 59, 85-111 (2001)

26. Mumford, D., Shah, J.: Optimal approximations by piecewise smooth functions and associated variational problems. Commun. Pure Appl. Math. 42(5), 577-685 (1989)

27. Osher, S., Solé, A., Vese, L.: Image decomposition and restoration using total variation minimization and the $H^{-1}$ norm. Multiscale Model. Simul. 1(3), 349-370 (2003)

28. Rockafellar, P.T.: Convex Analysis. Princeton Univ. Press, Princeton (1970)

29. Roudenko, S.: Noise and texture detection in image processing. Preprint (2004)

30. Rudin, L.I., Osher, S.: Total variation based image restoration with free local constraints. In: Proceedings of ICIP 1994, vol. 1, pp. 31-35 (1994)

31. Rudin, L., Osher, S., Fatemi, E.: Nonlinear total variation based noise removal algorithms. Physica D 60, 259-268 (1992)

32. Temam, R., Strang, G.: Functions of bounded deformation. Arch. Ration. Mech. Anal. 75(1), 7-21 (1980)

33. Tadmor, E., Nezzar, S., Vese, L.: A multiscale image representation using hierarchical $(B V, L 2)$ decompositions. Multiscale Model. Simul. 2(4), 554-579 (2004)

34. Starck, J.-L., Elad, M., Donoho, D.L.: Image decomposition: separation of texture from Piece-Wise Smooth content. In: SPIE Annual Meeting, San Diego, California, 3-8 August 2003

35. Vese, L.: A study in the BV space of a denoising-deblurring variational problem. Appl. Math. Optm. 44, 131-161 (2001)

36. Vese, L., Osher, S.: Modeling textures with total variation minimization and oscillating patterns in image processing. J. Sci. Comput. 19(1-3), 553-572 (2003) 